\title{
Electromagnetic Scattering at the Waveguide Step between Equilateral Triangular Waveguides
}

\author{
Ana Morán-López, ${ }^{1}$ Juan Córcoles, ${ }^{1}$ Jorge A. Ruiz-Cruz, ${ }^{1}$ \\ José R. Montejo-Garai, ${ }^{2}$ and Jesús M. Rebollar ${ }^{2}$ \\ ${ }^{1}$ Escuela Politécnica Superior, Universidad Autónoma de Madrid, C/Francisco Tomás y Valiente 11, 28049 Madrid, Spain \\ ${ }^{2}$ ETSI de Telecomunicación, Universidad Politécnica de Madrid, Avenida Complutense 30, 28040 Madrid, Spain
}

Correspondence should be addressed to Ana Morán-López; ana.moran@uam.es

Received 18 May 2016; Accepted 19 June 2016

Academic Editor: Remi Léandre

Copyright (C) 2016 Ana Morán-López et al. This is an open access article distributed under the Creative Commons Attribution License, which permits unrestricted use, distribution, and reproduction in any medium, provided the original work is properly cited.

\begin{abstract}
The analysis of the electromagnetic scattering at discontinuities between equilateral triangular waveguides is studied. The complete electromagnetic solution is derived using analytical closed form expressions for the mode spectrum of the equilateral waveguide. The mathematical formulation of the electromagnetic scattering problem is based on the quasi-analytical Mode-Matching method. This method benefits from the electromagnetic field division into symmetries as well as from the plane wave formulation presented for the expressions involved. The unification of the surface integrals used in the method thanks to the plane wave formulation is revealed, leading to expressions that are very well suited for its implementation in an electromagnetic analysis and design code. The obtained results for some cases of interest (building blocks for microwave components for communication systems) are verified using other numerical methods included in a commercial software package, showing the potential of the presented approach based on quasi-analytic expressions.
\end{abstract}

\section{Introduction}

Nowadays radiofrequency systems are widely used for very diverse applications. The GPS system for positioning, the LTE system for mobile communications, the DBS system for digital broadcasting, and ground penetrating radar or remote security screening are some examples of the usage of the electromagnetic spectrum that extends from some GHz to several hundred $\mathrm{GHz}[1,2]$. The increasing demand of the quality of service has led to very restrictive system specifications, which are translated into more complex microwave and millimeterwave devices. In order to accomplish such designs, CAD (Computer Aided Design) tools have experienced a strong development over the last decades $[3,4]$.

These tools solve Maxwell equations using numerical approaches or quasi-analytical methods [5]. Commercial tools as CST Microwave Studio or Ansys HFSS focus on the numerical solutions to Maxwell equations and develop methods as the Finite Element Method or the Finite Differences
Time Domain Method. This kind of methods has the advantage of being extremely flexible when coping with problems that involve arbitrary geometries. In contrast, they can be extremely time-consuming compared to the quasi-analytical methods. This paper focuses on the latter type of methods (i.e., quasi-analytical methods), particularly in the ModeMatching method [6]. This method was developed some decades ago and has been applied to classical waveguides with analytic modal spectrum such as the rectangular waveguide, the circular waveguide, and the elliptical waveguide for many types of waveguide devices such as transformers, filters, and couplers [7-11]. However, it has not been applied to the equilateral triangular waveguide yet.

This paper addresses the Mode-Matching method applied to the equilateral triangular geometry, giving a flexible mathematical formulation based on well-known plane waves. This formulation simplifies the surface integrals of the method and allows a very easy generalization to other problems involving waveguides whose modes can be written as a plane wave 
expansion. In order to validate the formulation, several case studies are presented and compared with the results given by other different numerical methods from commercial software.

\section{Modal Fields in the Equilateral Triangular Waveguide}

The electromagnetic field enclosed in an arbitrary cross section waveguide can be described as an orthogonal series of modes. When the considered transmission medium exhibits translation symmetry along the propagation axis the electric $(\vec{E})$ and magnetic $(\vec{H})$ field can be expressed through their transversal and longitudinal components [12-14]:

$$
\begin{aligned}
\vec{E}= & \sum_{p=1}^{\infty}\left(a_{p} e^{-\gamma_{p} z}+b_{p} e^{\gamma_{p} z}\right) \vec{e}_{p} \\
& +\left(a_{p} e^{-\gamma_{p} z}-b_{p} e^{\gamma_{p} z}\right) e_{z p} \widehat{z} \\
\vec{H}= & \sum_{p=1}^{\infty}\left(a_{p} e^{-\gamma_{p} z}-b_{p} e^{\gamma_{p} z}\right) \vec{h}_{p} \\
& +\left(a_{p} e^{-\gamma_{p} z}+b_{p} e^{\gamma_{p} z}\right) h_{z p} \widehat{z} .
\end{aligned}
$$

The terms $a_{p} e^{-\gamma_{p} z}$ and $b_{p} e^{\gamma_{p} z}$ are called the modal amplitudes at an arbitrary $z$ plane, of which the complex variables $a_{p}$ and $b_{p}$ are determined by the excitation and load of the waveguide. The propagation constant $\gamma_{p}$ and the transversal fields $\vec{e}_{p}$ and $\vec{h}_{p}$ of each mode depend on the geometry of the waveguides under consideration. The geometries of a classical circular waveguide and a classical rectangular waveguide are shown in Figures 1(a) and 1(b), respectively. In this paper the equilateral triangular waveguide, shown in Figure 1(c), is analyzed. As it is known, for uniform cross-section waveguides made up of a single enclosing metallic conductor filled with a homogeneous dielectric material, the three-dimensional Maxwell equations simplify to the scalar 2D Helmholtz equation $[13,14]$ :

$$
\Delta_{t} \phi_{p}+k_{c p}^{2} \phi_{p}=0
$$

which must be held in the inner cross section of the waveguide under consideration. From this function, directly related to the $z$-field component of the electric or magnetic field, the full electromagnetic field is obtained. The expressions of the modal components $\vec{e}_{p}, \vec{h}_{p}, e_{z p}$, and $h_{z p}$ in (1a)-(lb) are included in Appendix. The orthogonal modes that constitute the complete electromagnetic solution for a single conductor waveguide can be divided into two families: the so-called TE (Transversal Electric) modes and TM (Transversal Magnetic) modes. The former are calculated imposing homogeneous Neumann-type boundary conditions in (2) along the contour $C$ of the waveguide (see Figure 1(d)), whereas the latter are calculated imposing homogeneous Dirichlet-type boundary conditions:

$$
\begin{gathered}
\left.\frac{\partial \phi_{p}}{\partial n}\right|_{C}=0, \text { TE modes, } \\
\left.\phi_{p}\right|_{C}=0, \text { TM modes. }
\end{gathered}
$$

An initial characterization of the triangular waveguide can be found in $[15,16]$. The scalar function $\phi_{p}$ for the complete solution in an equilateral triangular waveguide is found in $[17,18]$, where the complete set of solutions are divided not only into TE and TM modes but also into symmetries with respect to one of its medians. These functions referring to the coordinate system selected in this paper (see Figure 1(d)), using superscript $S$ for symmetric solutions and $A$ for asymmetric solutions, are

$$
\begin{aligned}
\phi_{\mathrm{TE}}^{S}= & \cos \left(\widetilde{k}_{y 1}(y-b)\right) \cos \left(\widetilde{k}_{x 1} x\right) \\
& +\cos \left(\widetilde{k}_{y 2}(y-b)\right) \cos \left(\widetilde{k}_{x 2} x\right) \\
& +\cos \left(\widetilde{k}_{y 3}(y-b)\right) \cos \left(\widetilde{k}_{x 3} x\right), \\
\phi_{\mathrm{TE}}^{A}= & \cos \left(\widetilde{k}_{y 1}(y-b)\right) \sin \left(\widetilde{k}_{x 1} x\right) \\
& +\cos \left(\widetilde{k}_{y 2}(y-b)\right) \sin \left(\widetilde{k}_{x 2} x\right) \\
& +\cos \left(\widetilde{k}_{y 3}(y-b)\right) \sin \left(\widetilde{k}_{x 3} x\right), \\
\phi_{\mathrm{TM}}^{S}= & \sin \left(\widetilde{k}_{y 1}(y-b)\right) \cos \left(\widetilde{k}_{x 1} x\right) \\
& +\sin \left(\widetilde{k}_{y 2}(y-b)\right) \cos \left(\widetilde{k}_{x 2} x\right) \\
& +\sin \left(\widetilde{k}_{y 3}(y-b)\right) \cos \left(\widetilde{k}_{x 3} x\right), \\
\phi_{\mathrm{TM}}^{A}= & \sin \left(\widetilde{k}_{y 1}(y-b)\right) \sin \left(\widetilde{k}_{x 1} x\right) \\
& +\sin \left(\widetilde{k}_{y 2}(y-b)\right) \sin \left(\widetilde{k}_{x 2} x\right) \\
& +\sin \left(\widetilde{k}_{y 3}(y-b)\right) \sin \left(\widetilde{k}_{x 3} x\right),
\end{aligned}
$$

where

$$
\begin{aligned}
& \tilde{k}_{y 1}=\frac{(-m-n) \pi}{b}, \\
& \tilde{k}_{y 2}=\frac{m \pi}{b}, \\
& \tilde{k}_{y 3}=\frac{n \pi}{b}, \\
& \tilde{k}_{x 1}=\frac{(m-n) \pi}{\sqrt{3} b}, \\
& \tilde{k}_{x 2}=\frac{(2 n+m) \pi}{\sqrt{3} b}, \\
& \tilde{k}_{x 3}=\frac{(-2 m-n) \pi}{\sqrt{3} b} .
\end{aligned}
$$




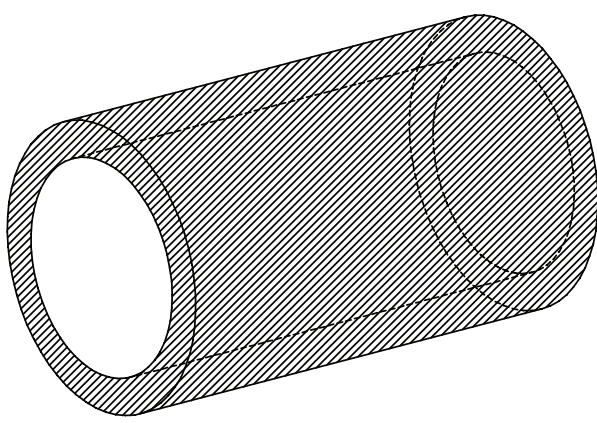

$\sigma=\infty$

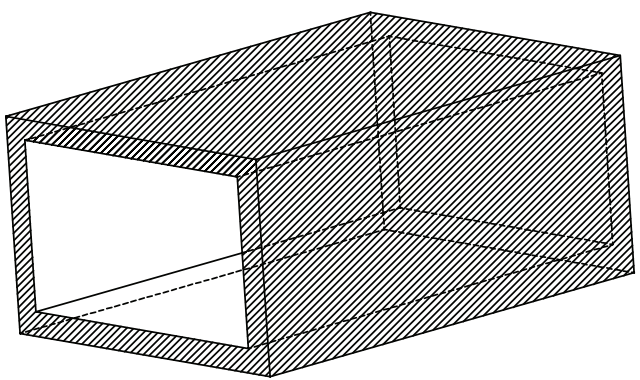

$\sigma=\infty$

(b)
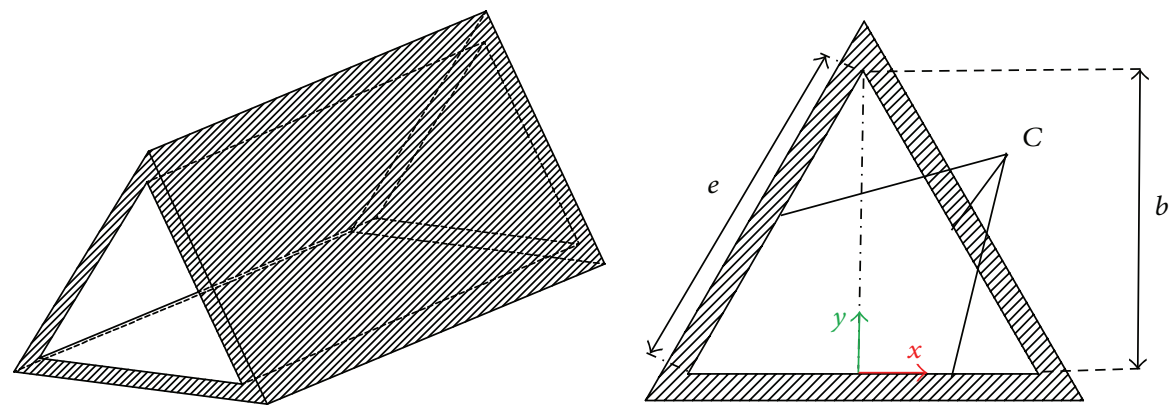

$\sigma=\infty$

(d)

FIGURE 1: (a) Classical circular waveguide, (b) classical rectangular waveguide, (c) equilateral triangular waveguide of this paper, and (d) reference coordinate system used. Conductivity $\sigma=\infty$ indicates a perfect electric wall boundary condition.

In the preceding definitions, variable $b$ in constants $\widetilde{k}_{y_{1,2,3}}$ and $\tilde{k}_{x_{1,2,3}}$ represents the height of the equilateral cross section of the waveguide. It is related to the side $e$ of the equilateral triangular waveguide through $b=e \sqrt{3} / 2$. Each modal solution that belongs to the series (1a)-(1b) is denoted by subindex $p\left(\vec{e}_{p}, \vec{h}_{p}\right)$, which identifies a single pair $(m, n)$ and one of the four families that have been introduced $\left(\mathrm{TE}^{S}, \mathrm{TE}^{A}\right.$, $\mathrm{TM}^{S}$, and $\mathrm{TM}^{A}$ ). $\mathrm{TE}_{m n}^{S}$ modes arise with subscripts $m \geq n \geq$ $0, \mathrm{TE}_{m n}^{A}$ modes with subscripts $m>n \geq 0, \mathrm{TM}_{m n}^{S}$ modes with $m \geq n>0$, and $\mathrm{TM}_{m n}^{A}$ modes with $m>n>0$. It can be seen that (4a)-(4b) fulfill homogeneous Neumann boundary conditions (3a) and that (4c)-(4d) fulfill homogeneous Dirichlet boundary conditions (3b). Finally, the cut-off wavenumber $k_{c p}$ in (2) can be calculated for each mode using

$$
k_{c p}=\frac{4 \pi}{3 e} \sqrt{m^{2}+n^{2}+m n},
$$

which shows that symmetric and asymmetric TE and TM modes are degenerated (share the same cut-off wavenumber value). It can be easily seen that the cut-off wavenumber $k_{c p}$ can also be derived as:

$$
k_{c p}^{2}=\tilde{k}_{x i}^{2}+\widetilde{k}_{y i}^{2}, \quad i=1,2,3 .
$$

The cut-off frequency associated with each particular mode, which indicates the minimum frequency where the propagation constant associated with that mode has null attenuation (i.e., it is purely imaginary) for lossless media $[13,14]$, is defined as

$$
f_{c p}=\frac{k_{c p}}{2 \pi \sqrt{\mu \varepsilon}},
$$

where $\mu$ is the magnetic permeability and $\varepsilon$ is the electric permittivity of the transmission medium filling the waveguide (being $(\sqrt{\mu \varepsilon})^{-1}=2.9979 \cdot 10^{8} \mathrm{~m} / \mathrm{s}$ for the vacuum). The fundamental modes (i.e., those with lowest cut-off frequency) are $\mathrm{TE}_{10}^{A}$ and $\mathrm{TE}_{10}^{S}$. The cut-off wavenumbers, the cut-off frequencies, and the associated families of the first fifty modes in a hollow waveguide of side $e=1 \mathrm{~cm}$ are given in Table 1 . This table can be used for any other equilateral triangular waveguide since $k_{c}$ and $f_{c}$ only need to be scaled by the new side $e^{\prime}$.

The electric and magnetic field patterns of the first twelve modes are shown in Figure 2. Electric fields (in blue arrows) and magnetic fields (in green arrows) are perpendicular to each other. Besides, the former are perpendicular to the sides of the equilateral triangle, whereas the latter are tangential, as it is required by the boundary conditions of a perfect conductor. Symmetric TE modes $\left(\mathrm{TE}_{m n}^{S}\right)$ and asymmetric $\mathrm{TM}$ 
TABLE 1: Cut-off wavenumbers, cut-off frequencies, and associated families for the first fifty modes in a hollow equilateral triangular waveguide of side $e=1 \mathrm{~cm}$.

\begin{tabular}{lccc}
\hline$(m, n)$ & $k_{c}\left[\mathrm{~cm}^{-1}\right]$ & $f_{c}[\mathrm{GHz}]$ & Mode designation \\
\hline$(1,0)$ & 4.189 & 19.99 & $\mathrm{TE}^{A}, \mathrm{TE}^{S}$ \\
$(1,1)$ & 7.255 & 34.62 & $\mathrm{TE}^{S}, \mathrm{TM}^{S}$ \\
$(2,0)$ & 8.378 & 39.97 & $\mathrm{TE}^{A}, \mathrm{TE}^{S}$ \\
$(2,1)$ & 11.082 & 52.88 & $\mathrm{TE}^{A}, \mathrm{TE}^{S}, \mathrm{TM}^{A}, \mathrm{TM}^{S}$ \\
$(3,0)$ & 12.566 & 59.96 & $\mathrm{TE}^{A}, \mathrm{TE}^{S}$ \\
$(2,2)$ & 14.510 & 69.23 & $\mathrm{TE}^{S}, \mathrm{TM}^{S}$ \\
$(3,1)$ & 15.103 & 72.06 & $\mathrm{TE}^{A}, \mathrm{TE}^{S}, \mathrm{TM}^{A}, \mathrm{TM}^{S}$ \\
$(4,0)$ & 16.755 & 79.94 & $\mathrm{TE}^{A}, \mathrm{TE}^{S}$ \\
$(3,2)$ & 18.259 & 87.12 & $\mathrm{TE}^{A}, \mathrm{TE}^{S}, \mathrm{TM}^{A}, \mathrm{TM}^{S}$ \\
$(4,1)$ & 19.195 & 91.59 & $\mathrm{TE}^{A}, \mathrm{TE}^{S}, \mathrm{TM}^{A}, \mathrm{TM}^{S}$ \\
$(5,0)$ & 20.944 & 99.93 & $\mathrm{TE}^{A}, \mathrm{TE}^{S}$ \\
$(3,3)$ & 21.766 & 103.85 & $\mathrm{TE}^{S}, \mathrm{TM}^{S}$ \\
$(4,2)$ & 22.165 & 105.76 & $\mathrm{TE}^{A}, \mathrm{TE}^{S}, \mathrm{TM}^{A}, \mathrm{TM}^{S}$ \\
$(5,1)$ & 23.322 & 111.28 & $\mathrm{TE}^{A}, \mathrm{TE}^{S}, \mathrm{TM}^{A}, \mathrm{TM}^{S}$ \\
$(6,0)$ & 25.133 & 119.92 & $\mathrm{TE}^{A}, \mathrm{TE}^{S}$ \\
$(4,3)$ & 25.479 & 121.57 & $\mathrm{TE}^{A}, \mathrm{TE}^{S}, \mathrm{TM}^{A}, \mathrm{TM}^{S}$ \\
$(5,2)$ & 26.159 & 124.81 & $\mathrm{TE}^{A}, \mathrm{TE}^{S}, \mathrm{TM}^{A}, \mathrm{TM}^{S}$ \\
\hline
\end{tabular}

modes $\left(\mathrm{TM}_{m n}^{A}\right)$ show perfect electric wall (PEW) symmetry with respect to $x=0$. On the other hand, asymmetric TE modes $\left(\mathrm{TE}_{m n}^{A}\right)$ and symmetric TM modes $\left(\mathrm{TM}_{m n}^{S}\right)$ show perfect magnetic wall (PMW) symmetry at $x=0$.

\section{Overview of the Mode-Matching Method}

The main goal of the Mode-Matching method is to solve the modal amplitudes of (1a)-(1b) and relate them through the Generalized Scattering Matrix (GSM) $[9,19]$, used to characterize the full response by cascading individual building blocks also in antenna problems $[20,21]$. In this section an overview of this method is introduced regardless of the cross section geometry of the waveguides involved in the analyzed discontinuity. In Section 4 this method will be particularized to our geometry of interest (i.e., the equilateral triangular waveguide).

The Mode-Matching method is based on the fact that the total transversal fields $\left(\vec{E}_{t}, \vec{H}_{t}\right)$ must be continuous across the transversal surface in the waveguide step under consideration. This surface is shown in Figure 3(a), where the waveguide $(w)$ has been ended with a perfect conductor wall except in the intersected area with the smaller waveguide $(s)$, and both waveguides are depicted without conductor thickness. According to (1a) and (1b), the transversal electromagnetic field at both sides of a step or discontinuity placed at the plane $z=0$ is represented by

$$
\begin{aligned}
& \left.\vec{E}_{t}^{(w)}\right|_{A_{w}, z=0^{-}}=\sum_{j=1}^{N_{w}}\left(a_{j}^{(w)}+b_{j}^{(w)}\right) \vec{e}_{j}^{(w)}, \\
& \left.\vec{E}_{t}^{(s)}\right|_{A_{s}, z=0^{+}}=\sum_{i=1}^{N_{s}}\left(b_{i}^{(s)}+a_{i}^{(s)}\right) \vec{e}_{i}^{(s)},
\end{aligned}
$$

$$
\begin{aligned}
& \left.\vec{H}_{t}^{(w)}\right|_{A_{w}, z=0^{-}}=\sum_{j=1}^{N_{w}}\left(a_{j}^{(w)}-b_{j}^{(w)}\right) \vec{h}_{j}^{(w)}, \\
& \left.\vec{H}_{t}^{(s)}\right|_{A_{s}, z=0^{+}}=\sum_{i=1}^{N_{s}}\left(b_{i}^{(s)}-a_{i}^{(s)}\right) \vec{h}_{i}^{(s)},
\end{aligned}
$$

where superscripts $(w)$ and $(s)$ refer to each involved waveguide (see Figure 3(b) for clarification). It is important to highlight that, in order to formulate an affordable computational problem, in the preceding equations the number of modes in each waveguide has been truncated to $N_{w}$ and $N_{s}$, respectively. The boundary conditions applied across the aperture interface $A_{s}$ that preserve the transversal fields are

$$
\begin{aligned}
& \text { EFBC in } A_{w}: \widehat{z} \times \vec{E}_{t}^{(w)}= \begin{cases}0, & \text { in } A_{c}, z=0 \\
\widehat{z} \times \vec{E}_{t}^{(s)} & \text { in } A_{s}, z=0\end{cases} \\
& \text { MFBC in } A_{s}: \hat{z} \times \vec{H}_{t}^{(w)}=\widehat{z} \times \vec{H}_{t}^{(s)} \text { in } \mathrm{A}_{s}, z=0 \text {, }
\end{aligned}
$$

where EFBC stands for Electric Field Boundary Condition and MFBC stands for Magnetic Field Boundary Condition. The boundary conditions are imposed using a Galerkin method, where the EFBC is tested with the modal magnetic fields of waveguide $(w)$ (i.e., $h_{j}^{(w)}$ ) and the MFBC is tested with the modal electric fields of waveguide $(s)$ (i.e., $\left.e_{i}^{(s)}\right)$ [11]. Arranging the modal amplitudes in column vectors $\left(\mathbf{a}_{g}=\left[\cdots a_{p}^{(g)} \cdots\right]^{T}\right.$, $\mathbf{b}_{g}=\left[\cdots b_{p}^{(g)} \cdots\right]^{T}$, being $\left.g=w, s\right)$, this method leads to the following system of linear equations:

$$
\begin{aligned}
\text { EFBC: }\left(\mathbf{a}_{w}+\mathbf{b}_{w}\right) & =\mathbf{X}^{t}\left(\mathbf{a}_{s}+\mathbf{b}_{s}\right) \quad\left(N_{w} \text { eqs. }\right), \\
\text { MFBC: } \mathbf{X}\left(\mathbf{a}_{w}-\mathbf{b}_{w}\right) & =\left(\begin{array}{ll}
\left.\mathbf{b}_{s}-\mathbf{a}_{s}\right) \quad\left(N_{s} \text { eqs. }\right),
\end{array}\right.
\end{aligned}
$$

where the term $\mathbf{X}$ is the following matrix:

$$
\mathbf{X}=\left[X_{i j}\right]=Y_{j}^{(w)} \iint_{A_{s}} \vec{e}_{i}^{(s)} \cdot \vec{e}_{j}^{(w)} d s
$$

The previous integral is expressed in terms of the electric field, but it is possible to use the magnetic field as well since $Y_{j}\left(\vec{e}_{i} \cdot \vec{e}_{j}\right)=Z_{i}\left(\vec{h}_{i} \cdot \vec{h}_{j}\right)$, where $Y_{j}$ and $Z_{i}$ stand for the mode admittance and the mode impedance, respectively, in Appendix. The relationship between the modal amplitudes obtained solving the previous linear equation system is given by the GSM of the discontinuity:

$$
\begin{aligned}
\mathbf{S} & =\left[\begin{array}{cc}
\mathbf{X}^{t} \mathbf{F X}-\mathbf{I}_{w} & \mathbf{X}^{t} \mathbf{F} \\
\mathbf{F X} & \mathbf{F}-\mathbf{I}_{s}
\end{array}\right], \\
{\left[\begin{array}{c}
\mathbf{b}_{w} \\
\mathbf{b}_{s}
\end{array}\right] } & =\mathbf{S}\left[\begin{array}{c}
\mathbf{a}_{w} \\
\mathbf{a}_{s}
\end{array}\right],
\end{aligned}
$$

where $\mathbf{F}=2\left(\mathbf{I}_{s}+\mathbf{X} \mathbf{X}^{t}\right)^{-1}$ and $\mathbf{I}$ is the identity matrix of the corresponding size $\left(N_{w}\right.$ or $\left.N_{s}\right)$. The analysis of two or more discontinuities or steps is carried out cascading GSM matrices as in $[10,11,19]$. 


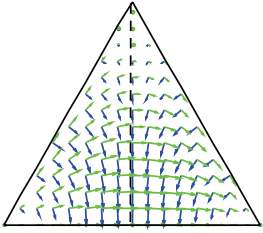

$\mathrm{TE}_{10}^{A}$

- - PMW

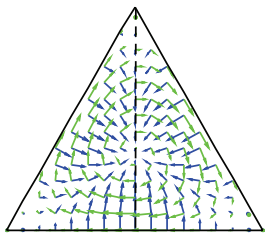

$\mathrm{TM}_{11}^{S}$

-. - PMW



$\mathrm{TE}_{21}^{A}$

-.- PMW

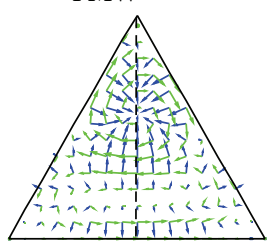

$\mathrm{TM}_{21}^{S}$

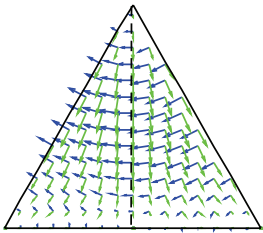

$\mathrm{TE}_{10}^{S}$

- - PEW

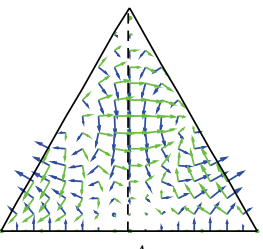

$\mathrm{TE}_{20}^{A}$

-.. PMW

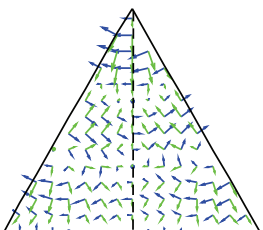

$\mathrm{TE}_{21}^{S}$
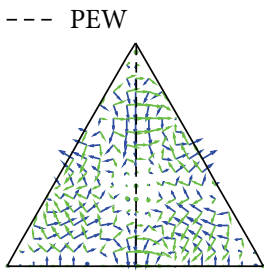

$\mathrm{TE}_{30}^{A}$

- - - PMW

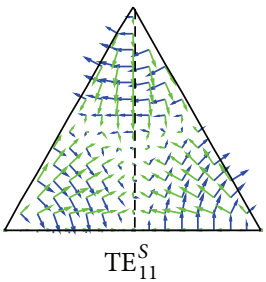

- - PEW

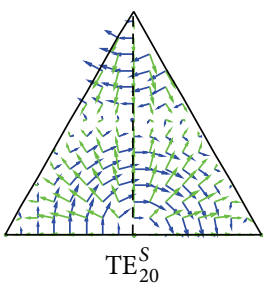

- - PEW

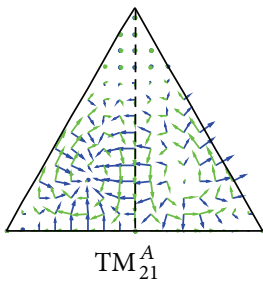

-.- PEW

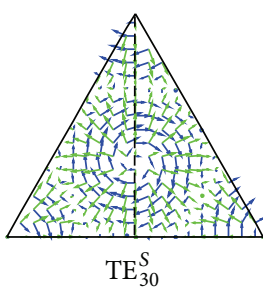

-. - PEW

FIGURE 2: Electric and magnetic field pattern (in blue and green arrows, resp.) for the first twelve modes in the equilateral triangular waveguide.

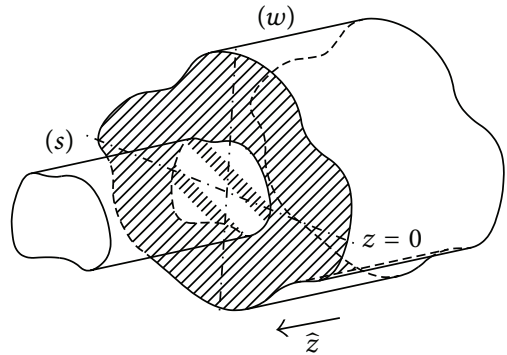

$A_{c}(\sigma=\infty)$

通 $A_{s}$

$A_{w}=A_{c} \cup A_{s}$ $(w)$



(b)

FIGURE 3: Generic waveguide step: (a) surface views and (b) modal amplitudes. 




$\mathbb{Z} A_{c}(\sigma=\infty)$

国 $A_{s}$

$A_{w}=A_{c} \cup A_{s}$

FigURE 4: Step between equilateral triangular waveguides.

\section{Mode-Matching Applied to Equilateral Triangular Steps}

Section 2 presented the basis functions $\phi_{\mathrm{TE}, \mathrm{TM}}^{S / A}$ needed to formulate the modal fields in any equilateral triangular waveguide. The modal fields $\vec{e}_{p}^{(g)}$ or $\vec{h}_{p}^{(g)}$ are required to compute the inner cross product matrix $\mathbf{X}$ seen in Section 3, where the Mode-Matching method was introduced. This section addresses the resolution of the surface integrals associated with the inner cross products and aims at providing a suitable formulation to solve discontinuities where one of the waveguide surfaces is completely included into the other one (see Figure 4). Towards that end, the functions given in (4a)-(4d) must be rewritten in a more convenient form based on plane waves:

$$
\phi_{\mathrm{TE} / \mathrm{TM}}^{\mathrm{S}, A / S, A}=\sum_{u=1}^{12} A_{u} e^{j\left(k_{x u} x+k_{y u} y\right)} .
$$

The resulting parameters $A_{u}, k_{x u}$, and $k_{y u}$ of the preceding equation are given in Table 2 . In order to highlight the benefits of the mathematical formulation used in this paper, it is sometimes convenient to define the wavenumber vector $\vec{k}_{u}=k_{x u} \hat{x}+k_{y u} \hat{y}$ and the position vector $\vec{r}=x \hat{x}+y \hat{y}$ and then rewrite $\vec{r} \cdot \vec{k}_{u}=k_{x u} x+k_{y u} y$.

Once the functions of the four mode families are formulated using (14), the surface integrals involved in the ModeMatching method are greatly simplified. The first surface integral that must be solved is the one associated with the normalization constant $N_{p}$ (see Appendix). This surface integral is solved in $[17,18]$ and its values are directly given by

$$
N_{p}= \begin{cases}\frac{16}{3 \sqrt{3} e^{2} k_{c p}^{2}} & \text { if } m \neq n \text { and } n \neq 0 \\ \frac{8}{3 \sqrt{3} e^{2} k_{c p}^{2}} & \text { if } m=n \text { or } n=0 .\end{cases}
$$

The other surface integrals that must be solved in the Mode-Matching method are those needed to calculate the inner cross product matrix $(\mathbf{X})$. The electric fields in (12)
TABLE 2: Values of parameters $k_{x u}, k_{y u}$, and $A_{u}$, where the parameters denoted as $\widetilde{k}_{y_{1,2,3}}$ and $\widetilde{k}_{x_{1,2,3}}$ are the ones described immediately after (4a)-(4d), and $\Psi$ and $\Omega$ are defined in Table 3.

\begin{tabular}{lccc}
\hline$u$ & $k_{x u}$ & $k_{y u}$ & $A_{u}$ \\
\hline 1 & $\widetilde{k}_{x 1}$ & $\widetilde{k}_{y 1}$ & $(1 / 4) e^{j\left(-\widetilde{k}_{y 1} b-\Psi-\Omega\right)}$ \\
2 & $-\widetilde{k}_{x 1}$ & $\widetilde{k}_{y 1}$ & $(1 / 4) e^{j\left(-\widetilde{k}_{y 1} b-\Psi+\Omega\right)}$ \\
3 & $\widetilde{k}_{x 1}$ & $-\widetilde{k}_{y 1}$ & $(1 / 4) e^{j\left(\widetilde{k}_{y 1} b+\Psi-\Omega\right)}$ \\
4 & $-\widetilde{k}_{x 1}$ & $-\widetilde{k}_{y 1}$ & $(1 / 4) e^{j\left(\widetilde{k}_{y 1} b+\Psi+\Omega\right)}$ \\
5 & $\widetilde{k}_{x 2}$ & $\widetilde{k}_{y 2}$ & $(1 / 4) e^{j\left(-\widetilde{k}_{y 2} b-\Psi-\Omega\right)}$ \\
6 & $-\widetilde{k}_{x 2}$ & $\widetilde{k}_{y 2}$ & $(1 / 4) e^{j\left(-\widetilde{k}_{y 2} b-\Psi+\Omega\right)}$ \\
7 & $\widetilde{k}_{x 2}$ & $-\widetilde{k}_{y 2}$ & $(1 / 4) e^{j\left(\widetilde{k}_{y 2} b+\Psi-\Omega\right)}$ \\
8 & $-\widetilde{k}_{x 2}$ & $-\widetilde{k}_{y 2}$ & $(1 / 4) e^{j\left(\widetilde{k}_{y 2} b+\Psi+\Omega\right)}$ \\
9 & $\widetilde{k}_{x 3}$ & $\widetilde{k}_{y 3}$ & $(1 / 4) e^{j\left(-\widetilde{k}_{y 3} b-\Psi-\Omega\right)}$ \\
10 & $-\widetilde{k}_{x 3}$ & $\widetilde{k}_{y 3}$ & $(1 / 4) e^{j\left(-\widetilde{k}_{y 3} b-\Psi+\Omega\right)}$ \\
11 & $\widetilde{k}_{x 3}$ & $-\widetilde{k}_{y 3}$ & $(1 / 4) e^{j\left(\widetilde{k}_{y 3} b+\Psi-\Omega\right)}$ \\
12 & $-\widetilde{k}_{x 3}$ & $-\widetilde{k}_{y 3}$ & $(1 / 4) e^{j\left(\widetilde{k}_{y 3} b+\Psi+\Omega\right)}$ \\
\hline
\end{tabular}

TABLE 3: Values of parameters $\Psi$ and $\Omega$ for Table 2 .

\begin{tabular}{lcc}
\hline Mode designation & $\Psi$ & $\Omega$ \\
\hline $\mathrm{TE}^{S}$ & 0 & 0 \\
$\mathrm{TE}^{A}$ & 0 & $\pi / 2$ \\
$\mathrm{TM}^{S}$ & $\pi / 2$ & 0 \\
$\mathrm{TM}^{A}$ & $\pi / 2$ & $\pi / 2$ \\
\hline
\end{tabular}

are computed according to Appendix, using the transversal gradient of the function in (14). Each term $X_{i j}$ of the inner cross product matrix $\mathbf{X}$ is computed as

$$
X_{i j}=\left(X_{i j, x}+X_{i j, y}\right) \frac{\sqrt{Y_{j}^{(w)}}}{\sqrt{Y_{i}^{(s)}}},
$$

where the terms $X_{i j, x}$ and $X_{i j, y}$ are independent of the frequency and the material filling the waveguide and refer to the surface integrals associated with the transversal fields in $\widehat{x}$ and $\hat{y}$ :

$$
\begin{aligned}
& X_{i j, x}=\sum_{r=1}^{12} \sum_{q=1}^{12} A_{s r}^{(s)} A_{s q}^{(w)} \iint_{A_{s}} e^{j\left(k_{x r}^{(s)}+k_{x q}^{(w)}\right) x} e^{j\left(k_{y r}^{(s)}+k_{y q}^{(w)}\right) y} d s, \\
& X_{i j, y}=\sum_{r=1}^{12} \sum_{q=1}^{12} A_{t r}^{(s)} A_{t q}^{(w)} \iint_{A_{s}} e^{j\left(k_{x r}^{(s)}+k_{x q}^{(w)}\right) x} e^{j\left(k_{y r}^{(s)}+k_{y q}^{(w)}\right) y} d s .
\end{aligned}
$$

In the above expressions $A_{s}$ is the intersected area between both equilateral waveguides as it is shown in Figure 4 . The variables $k_{x r}, k_{x q}, k_{y r}$, and $k_{y q}$ are the wavenumbers in (14), for each respective waveguide. Only the new amplitudes $A_{s r}, A_{s q}, A_{t r}$, and $A_{t q}$ must be computed according to the involved gradients shown in Appendix. The results of these amplitudes are given in Table 4. 
TABLE 4: Values of parameters $A_{s r}, A_{s q}, A_{t r}$, and $A_{t q}$.

\begin{tabular}{lcccc}
\hline$r, q$ & $A_{s r, s q}[\mathrm{TE}]$ & $A_{s r, s q}[\mathrm{TM}]$ & $A_{t r, t q}[\mathrm{TE}]$ & $A_{t r, t q}[\mathrm{TM}]$ \\
\hline 1 & $j \widetilde{k}_{y 1} A_{u}$ & $j \widetilde{k}_{x 1} A_{u}$ & $-j \widetilde{k}_{x 1} A_{u}$ & $j \widetilde{k}_{y 1} A_{u}$ \\
2 & $j \widetilde{k}_{y 1} A_{u}$ & $-j \widetilde{k}_{x 1} A_{u}$ & $j \widetilde{k}_{x 1} A_{u}$ & $j \widetilde{k}_{y 1} A_{u}$ \\
3 & $-j \widetilde{k}_{y 1} A_{u}$ & $j \widetilde{k}_{x 1} A_{u}$ & $-j \widetilde{k}_{x 1} A_{u}$ & $-j \widetilde{k}_{y 1} A_{u}$ \\
4 & $-j \widetilde{k}_{y 1} A_{u}$ & $-j \widetilde{k}_{x 1} A_{u}$ & $j \widetilde{k}_{x 1} A_{u}$ & $-j \widetilde{k}_{y 1} A_{u}$ \\
5 & $j \widetilde{k}_{y 2} A_{u}$ & $j \widetilde{k}_{x 2} A_{u}$ & $-j \widetilde{k}_{x 2} A_{u}$ & $j \widetilde{k}_{y 2} A_{u}$ \\
6 & $j \widetilde{k}_{y 2} A_{u}$ & $-j \widetilde{k}_{x 2} A_{u}$ & $j \widetilde{k}_{x 2} A_{u}$ & $j \tilde{k}_{y 2} A_{u}$ \\
7 & $-j \widetilde{k}_{y 2} A_{u}$ & $j \widetilde{k}_{x 2} A_{u}$ & $-j \widetilde{k}_{x 2} A_{u}$ & $-j \widetilde{k}_{y 2} A_{u}$ \\
8 & $-j \widetilde{k}_{y 2} A_{u}$ & $-j \widetilde{k}_{x 2} A_{u}$ & $j \widetilde{k}_{x 2} A_{u}$ & $-j \widetilde{k}_{y 2} A_{u}$ \\
9 & $j \widetilde{k}_{y 3} A_{u}$ & $j \widetilde{k}_{x 3} A_{u}$ & $-j \widetilde{k}_{x 3} A_{u}$ & $j j \tilde{k}_{y 3} A_{u}$ \\
10 & $j \widetilde{k}_{y 3} A_{u}$ & $-j \widetilde{k}_{x 3} A_{u}$ & $j \widetilde{k}_{x 3} A_{u}$ & $j \widetilde{k}_{y 3} A_{u}$ \\
11 & $-j \widetilde{k}_{y 3} A_{u}$ & $j \widetilde{k}_{x 3} A_{u}$ & $-j \widetilde{k}_{x 3} A_{u}$ & $-j \widetilde{k}_{y 3} A_{u}$ \\
12 & $-j \widetilde{k}_{y 3} A_{u}$ & $-j \widetilde{k}_{x 3} A_{u}$ & $j \widetilde{k}_{x 3} A_{u}$ & $-j \widetilde{k}_{y 3} A_{u}$ \\
\hline & & & &
\end{tabular}

These inner cross products can be arranged according to the families to which the modes in (12) belong, with matrix $\mathbf{X}$ then becoming a set of submatrices:

$$
\begin{aligned}
& \mathbf{X} \\
& =\left[\begin{array}{llll}
\mathbf{X}_{\mathrm{TE}^{S}-\mathrm{TE}}^{(s)-(w)} & \mathbf{X}_{\mathrm{TE}^{S}-\mathrm{TE}^{A}}^{(s)-(w)} & \mathbf{X}_{\mathrm{TE}^{S}-\mathrm{TM}^{S}}^{(s)-(w)} & \mathbf{X}_{\mathrm{TE}^{S}-\mathrm{TM}^{A}}^{(s)-(w)} \\
\mathbf{X}_{\mathrm{TE}^{A}-\mathrm{T}}^{(s)-(w)} & \mathbf{X}_{\mathrm{TE}^{A}-\mathrm{TE}^{A}}^{(s)-(w)} & \mathbf{X}_{\mathrm{TE}^{(s)}-(w)} & \mathbf{X}_{\mathrm{TE}^{A}-\mathrm{TM}^{A}}^{(s)-(w)} \\
\mathbf{X}_{\mathrm{TM}^{S}-\mathrm{TE}^{S}-(w)}^{(s)} & \mathbf{X}_{\mathrm{TM}^{S}-\mathrm{TE}^{A}}^{(s)-(w)} & \mathbf{X}_{\mathrm{TM}^{S}-\mathrm{TM}^{S}}^{(s)-(w)} & \mathbf{X}_{\mathrm{TM}^{S}-\mathrm{TM}^{A}}^{(s)-(w)} \\
\mathbf{X}_{\mathrm{TM}^{(s)-(w)}}^{(s)} & \mathbf{X}_{\mathrm{TM}^{(s)}-\mathrm{TE}^{A}}^{(s)} & \mathbf{X}_{\mathrm{TM}^{A}-\mathrm{TM}^{S}}^{(s)-(w)} & \mathbf{X}_{\mathrm{TM}^{A}-\mathrm{TM}^{A}}^{(s)-(w)}
\end{array}\right] .
\end{aligned}
$$

Some of the surface integrals associated with these submatrices can be discarded since their values are zero [22]. Therefore (18) becomes

$$
\mathbf{X}=\left[\begin{array}{ccll}
\mathbf{X}_{\mathrm{TE}^{S}-\mathrm{TE}^{S}}^{(s)-(w)} & \mathbf{X}_{\mathrm{TE}^{S}-\mathrm{TE}^{A}}^{(s)-(w)} & \mathbf{X}_{\mathrm{TE}^{S}-\mathrm{TM}^{S}}^{(s)-(w)} & \mathbf{X}_{\mathrm{TE}^{S}-\mathrm{TM}^{A}}^{(s)-(w)} \\
\mathbf{X}_{\mathrm{TE}^{(s)}-\mathrm{TE}^{S}}^{(w)} & \mathbf{X}_{\mathrm{TE}^{(s)}-\mathrm{TE}^{A}}^{(s)} & \mathbf{X}_{\mathrm{TE}^{A}-\mathrm{TM}^{S}}^{(s)-(w)} & \mathbf{X}_{\mathrm{TE}^{(s)}-\mathrm{TM}^{A}}^{(s)} \\
0 & 0 & \mathbf{X}_{\mathrm{TM}^{S}-\mathrm{TM}^{S}}^{(s)-(w)} & \mathbf{X}_{\mathrm{TM}^{S}-\mathrm{TM}^{A}}^{(s)-(w)} \\
0 & 0 & \mathbf{X}_{\mathrm{TM}^{A}-\mathrm{TM}^{S}}^{(s)-(w)} & \mathbf{X}_{\mathrm{TM}^{A}-\mathrm{TM}^{A}}^{(s)-(w)}
\end{array}\right]
$$

Moreover, having the TE and TM modes divided into symmetries allows discarding even more surface integrals when both waveguides have their symmetry axis aligned as in Figure 4. In that case, symmetric TE modes do not excite asymmetric TE modes, and symmetric TM modes do not excite asymmetric TM modes, nor does this happen in TE and TM modes of different perfect walls. Thus, in those conditions (19) can be simplified to

$$
\mathbf{X}=\left[\begin{array}{cccc}
\mathbf{X}_{\mathrm{TE}^{S}-\mathrm{TE}^{S}}^{(s)-(w)} & 0 & 0 & \mathbf{X}_{\mathrm{TE}^{S}-\mathrm{TM}^{A}}^{(s)-(w)} \\
0 & \mathbf{X}_{\mathrm{TE}^{A}-\mathrm{TE}^{A}}^{(s)-(w)} & \mathbf{X}_{\mathrm{TE}^{A}-\mathrm{TM}^{S}}^{(s)-(w)} & 0 \\
0 & 0 & \mathbf{X}_{\mathrm{TM}^{S}-\mathrm{TM}^{S}}^{(s)-(w)} & 0 \\
0 & 0 & 0 & \mathbf{X}_{\mathrm{TM}^{A}-\mathrm{TM}^{A}}^{(s)-(w)}
\end{array}\right]
$$

One significant advantage of the mathematical formulation used in this paper to describe the problem of the



Figure 5: Change in the coordinate system.

electromagnetic scattering between equilateral triangular waveguides is that any offset introduced in the reference coordinate system results again in plane waves with the same wavenumber vector and just a change in their associated amplitudes. Figure 5 shows an arbitrary offset between two coordinate systems. According to this representation, the basis functions in (14) referring to the new coordinate system are

$$
\phi_{\mathrm{TE} / \mathrm{TM}}^{\prime S, A / S, A}=\sum_{u=1}^{12} A_{u} e^{j\left(k_{x u}\left(x^{\prime}+\Delta x\right)+k_{y u}\left(y^{\prime}+\Delta y\right)\right)},
$$

which can be rewritten using the same parameters $k_{x u}$ and $k_{y u}$ already calculated in (14) and therefore introducing a simple change in its amplitudes:

$$
\phi_{\mathrm{TE} / \mathrm{TM}}^{\prime S, A / S, A}=\sum_{u=1}^{12} A_{u}^{\prime} e^{j\left(k_{x u} x^{\prime}+k_{y u} y^{\prime}\right)},
$$

where $A_{u}^{\prime}=A_{u} e^{j\left(k_{x u} \Delta x+k_{y u} \Delta y\right)}$. Another advantage of this formulation is that all the surface integrals involved in the calculation of the inner cross product matrix $\mathbf{X}$ can be reduced to the following simple exponential integral:

$$
I=\iint_{A_{s}} e^{j B_{1} x} e^{j B_{2} y} d s,
$$

where $B_{1}$ and $B_{2}$ are related to the additions of the corresponding wavenumbers and $A_{s}$ is the inner surface of the triangular intersection.

\section{Results and Discussion}

In order to prove the efficiency of the formulation presented in this paper, three cases are solved. For each case, the GSM is compared at different frequencies with the results given by the commercial software CST Microwave Studio. It is important to bear in mind that the modal series that describe the electromagnetic field in each waveguide must be truncated to a finite number of modes. Nevertheless, it is not advisable to use a very high number of modes from the beginning since it increases the computational cost and therefore the efficiency of the Mode-Matching technique is compromised. Besides, in this scenario, where two modal series have been matched, the problem of relative convergence arises. 


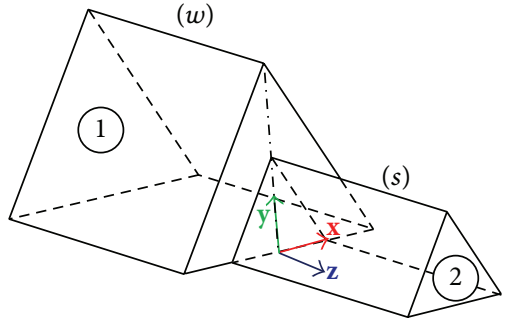

(a)

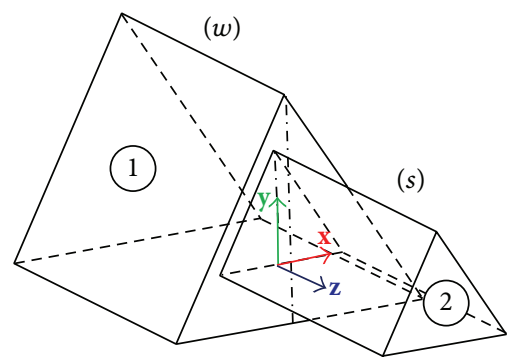

(b)

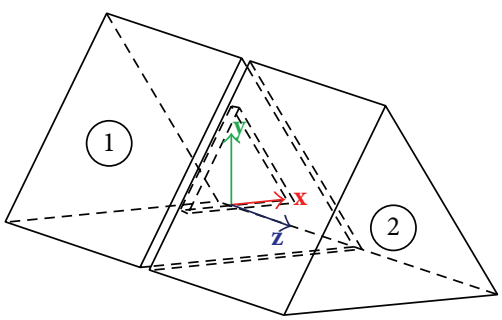

(c)

FIGURE 6: Case studies: single step (a) without offset and (b) with offset and (c) double step with offset.
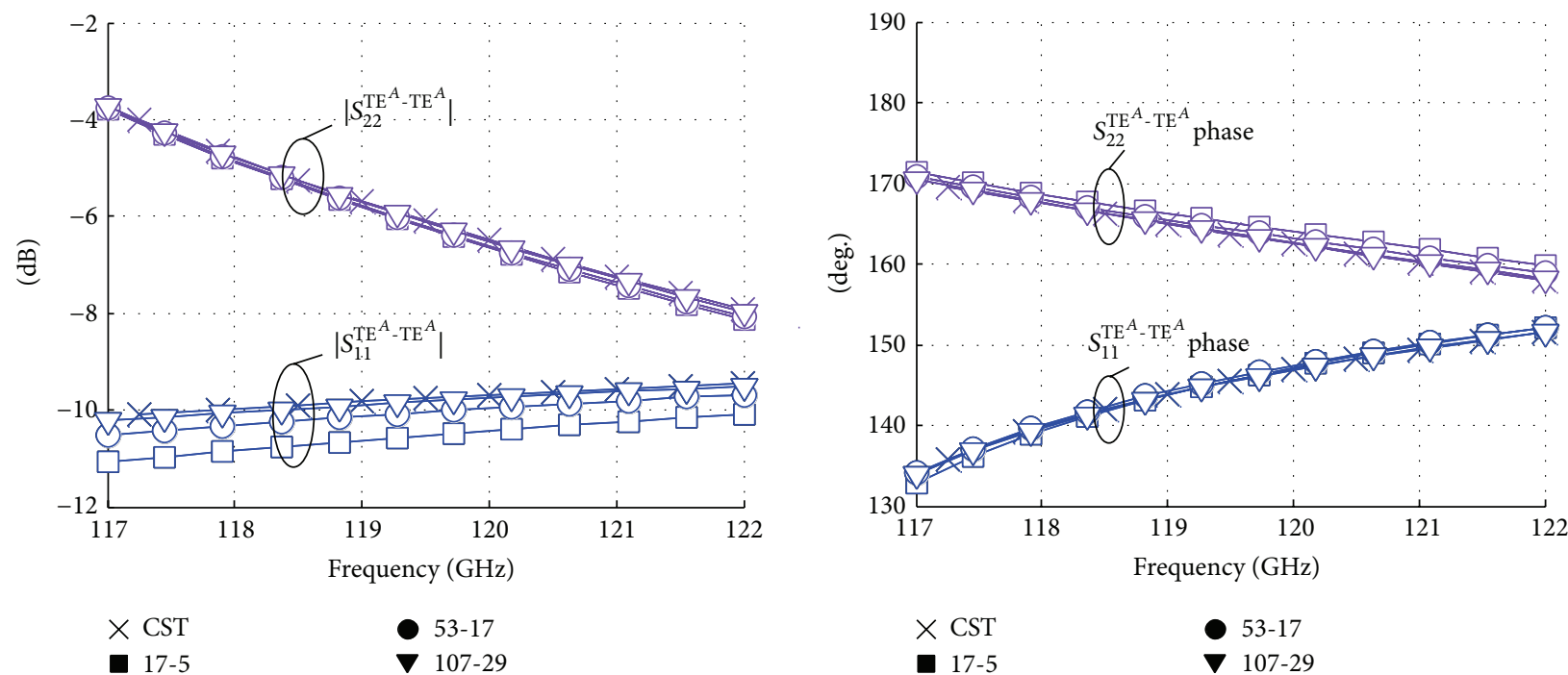

(a)

(b)



(c)



(d)

FIGURE 7: Case study a: mode $\mathrm{TE}_{10}^{A}$ in waveguide 1 to mode $\mathrm{TE}_{10}^{A}$ in waveguide 2: (a) reflection magnitude, (b) reflection phase, (c) transmission magnitude, and (d) transmission phase. 


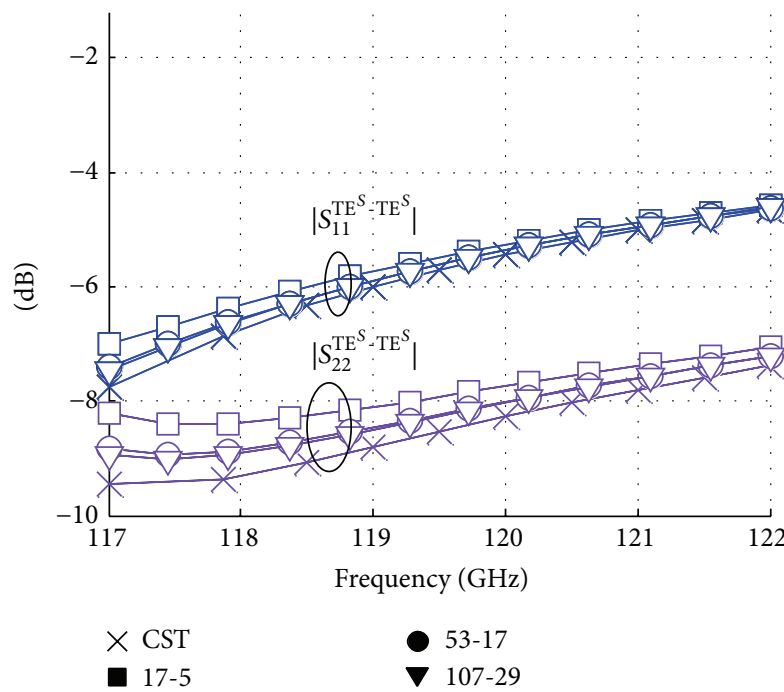

(a)



(c)

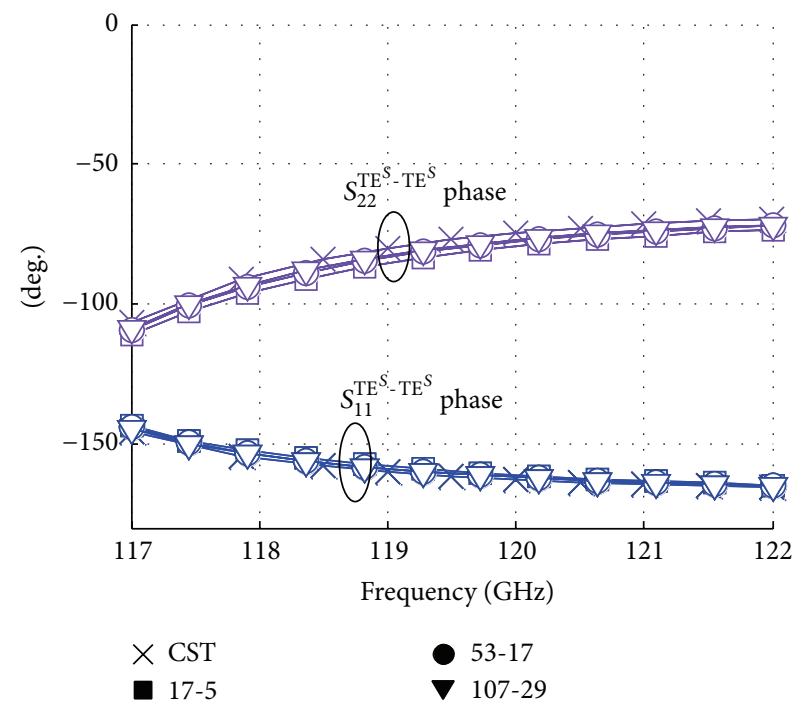

(b)

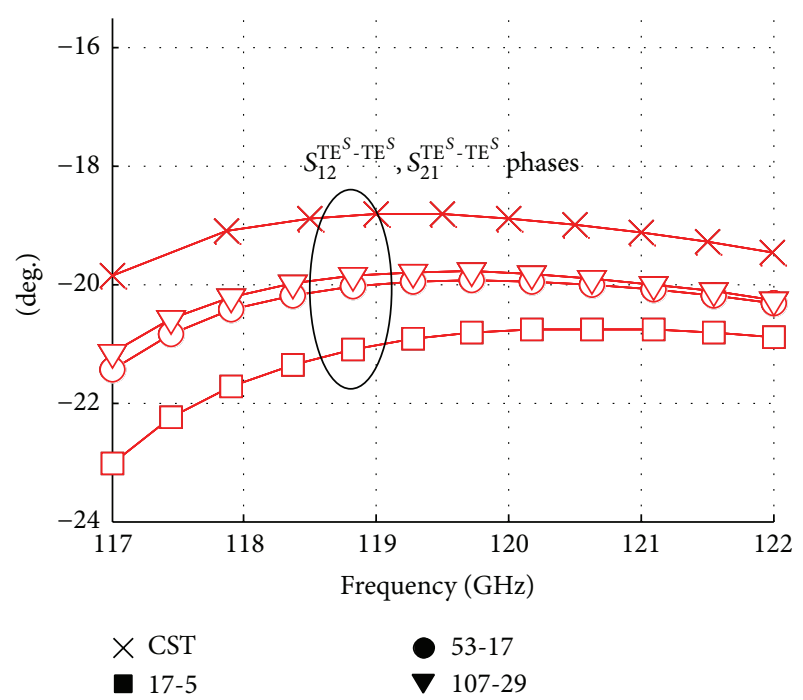

(d)

FIGURE 8: Case study a: mode $\mathrm{TE}_{10}^{S}$ in waveguide 1 to mode $\mathrm{TE}_{10}^{S}$ in waveguide 2: (a) reflection magnitude, (b) reflection phase, (c) transmission magnitude, and (d) transmission phase.

One of the well-known strategies to address the problem is to set a reference frequency $f_{c}^{\text {ref }}$ and select the modes whose cut-off frequency is below it in each waveguide. The reference frequency $f_{c}^{\text {ref }}$ is increased until the results decrease their variation with the number of selected modes. It will be seen that at that point they show a good agreement with the commercial software CST Microwave Studio.

According to the selected stopping criterion based on a cut-off frequency threshold, three pairs of mode relationships are used: 34-10, 106-34, and 214-58, where the former number indicates the number of modes considered in the largest waveguide and the latter the ones considered in the smallest one. The three case studies analyzed are depicted in Figure 6.
5.1. Single Step between Aligned Waveguides. In the first case study a single step between two equilateral triangular waveguides is considered, where both of them are centered at our coordinate reference system (see Figure 6(a)). The side of the waveguide $(s)$ is $\sqrt{3} \mathrm{~mm}$, which is port 2 in the generalized scattering parameters shown later on, whereas the side of the waveguide $(w)$, which is port 1 , is $2 \sqrt{3} \mathrm{~mm}$. Figures 7 and 8 show the comparison of the two first degenerated modes $\left(\mathrm{TE}_{10}^{S}\right.$ and $\left.\mathrm{TE}_{10}^{A}\right)$ using the presented formulation for the Mode-Matching method and the commercial software CST Microwave Studio.

The frequency range (i.e., $117-122 \mathrm{GHz}$ ) is selected in order to ensure the propagation of the pair of fundamental 


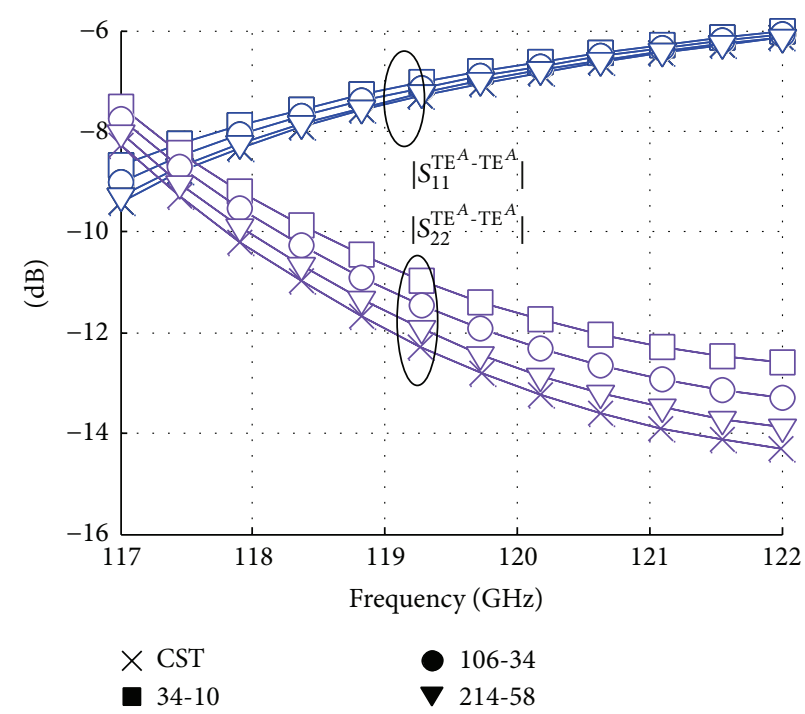

(a)

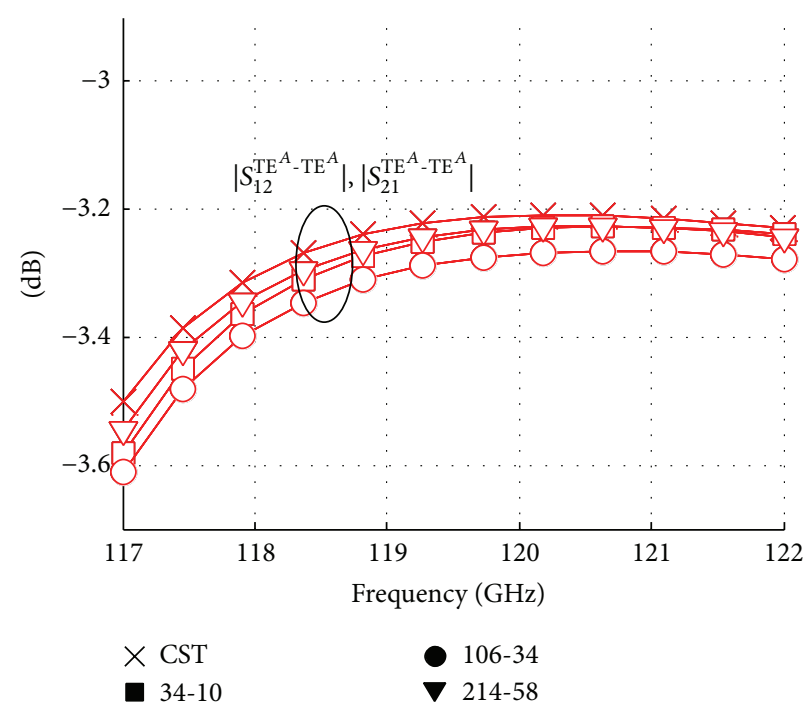

(c)

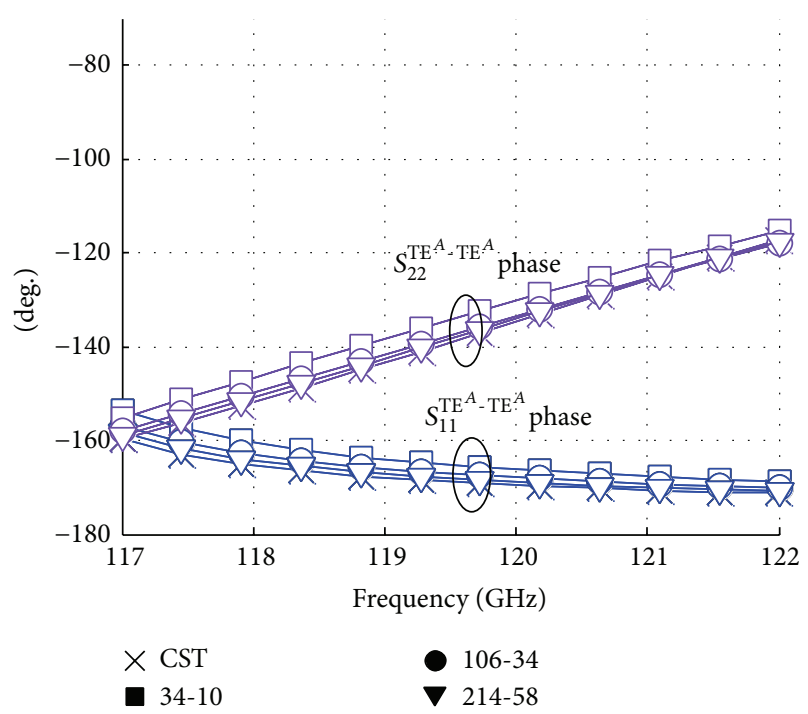

(b)



(d)

Figure 9: Case study b: mode $\mathrm{TE}_{10}^{A}$ in waveguide 1 to mode $\mathrm{TE}_{10}^{A}$ in waveguide 2: (a) reflection magnitude, (b) reflection phase, (c) transmission magnitude, and (d) transmission phase.

modes in each waveguide and guarantee a better comparison with the commercial software.

Figures $7(\mathrm{a})$ and $7(\mathrm{~b})$ show the magnitude and phase of the reflection parameters of the $\mathrm{TE}_{10}^{A}$ mode, that is, the relationship between the amplitudes of the incident and the reflected $\mathrm{TE}_{10}^{A}$ mode in each waveguide. Figures $7(\mathrm{c})$ and 7 (d) represent the magnitude and phase of the transmission parameters of the $\mathrm{TE}_{10}^{A}$ mode, that is, the relationship between the amplitudes of the same type of mode going through both waveguides.

In this case the energy transfer between the $\mathrm{TE}_{10}^{A}$ mode and the $\mathrm{TE}_{10}^{S}$ mode is not represented, since it is known in advance that this transference is zero due to the dual symmetries that these modes present. For the same reason, thanks to the division of the modes into symmetries the number of modes used is half the number used in the two following cases, leading to a faster convergence.

Figure 8 follows the same scheme with the mode $\mathrm{TE}_{10}^{S}$. It can be seen that, compared to the $\mathrm{TE}_{10}^{A}$ mode, the $\mathrm{TE}_{10}^{s}$ mode requires more modes in the series to achieve the results given by the numerical method in CST in general. In the $\mathrm{TE}_{10}^{A}$ mode only the $\left|S_{11}\right|$ parameter and the phase of the transmission parameters require a larger number of modes to converge.

5.2. Single Step between Misaligned Waveguides. In the second case study the previous waveguides are selected again, but a vertical offset of $0.08 \mathrm{~mm}$ and a horizontal offset of $0.02 \mathrm{~mm}$ are introduced (see Figure 6(b)). Figures 9-11 


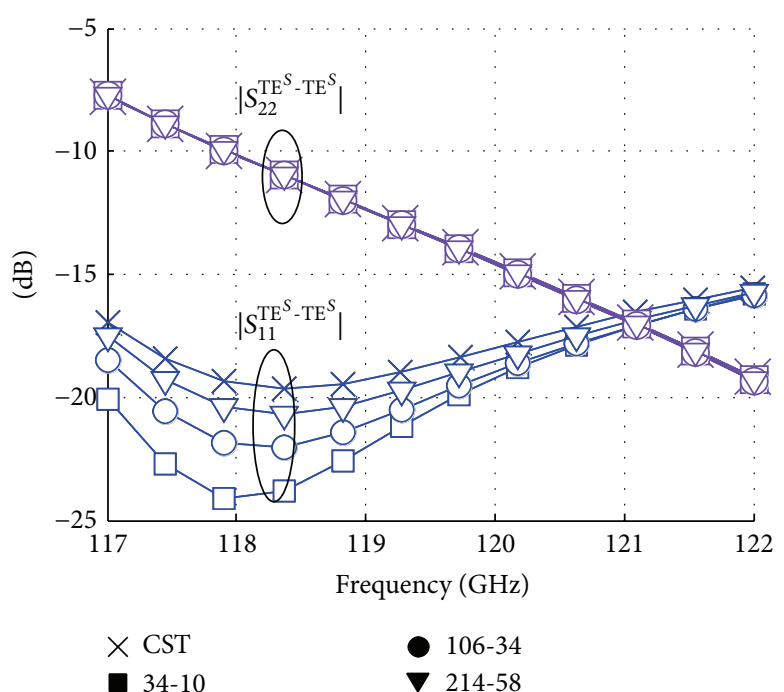

(a)

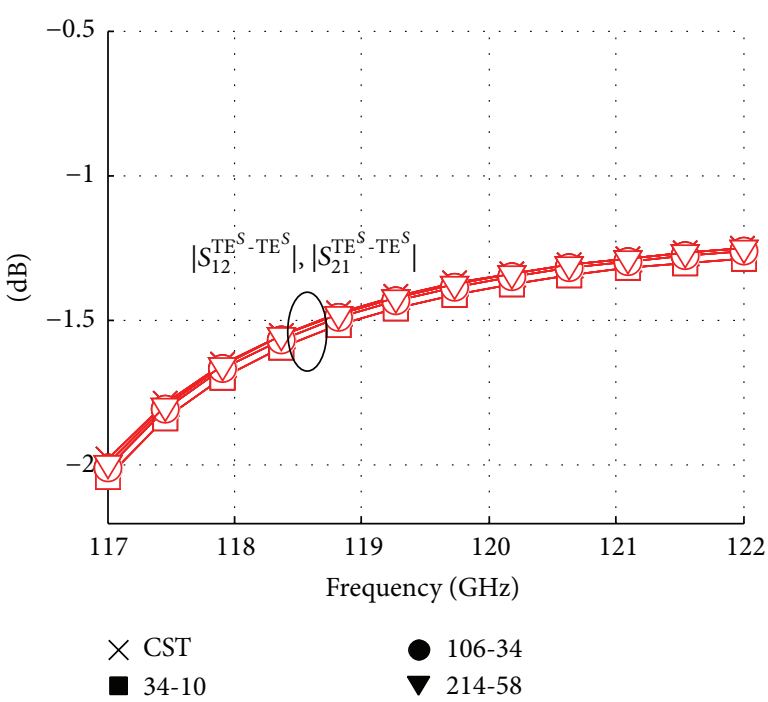

(c)

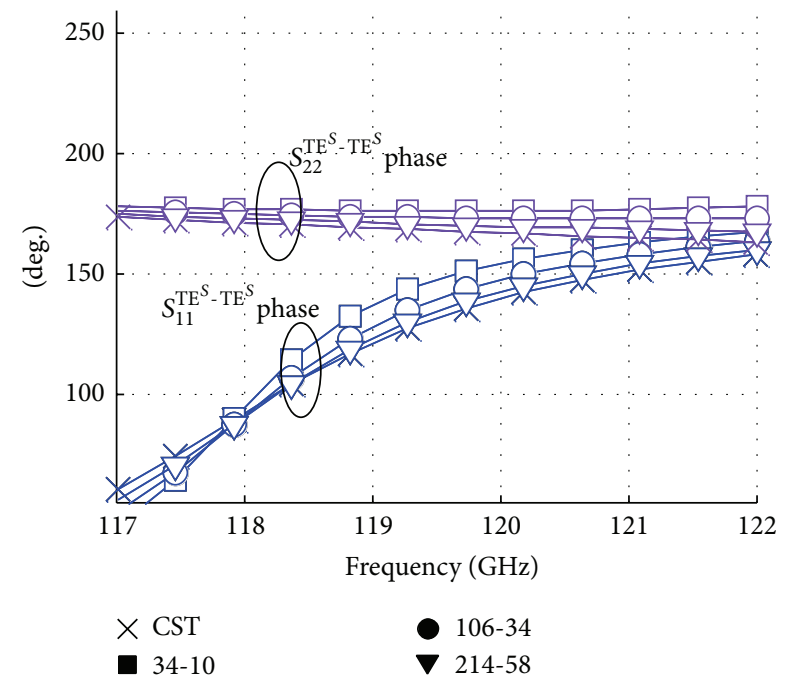

(b)



(d)

Figure 10: Case study b: mode $\mathrm{TE}_{10}^{S}$ in waveguide 1 to mode $\mathrm{TE}_{10}^{S}$ in waveguide 2: (a) reflection magnitude, (b) reflection phase, (c) transmission magnitude, and (d) transmission phase.

depict the results obtained using both the Mode-Matching technique and the CST Microwave Studio solver again for the two fundamental degenerate modes.

Figures 9 and 10 show that in this case both the $\mathrm{TE}_{10}^{A}$ and the $\mathrm{TE}_{10}^{S}$ modes require a higher number of modes to be similar to the results obtained by the CST commercial software.

It is interesting to include the energy transfer between the modes of different families, which is nonzero in this case study since the symmetry axis between the considered waveguides is no longer aligned. Figure 11 represents the relationships between the modal amplitudes of mode $\mathrm{TE}_{10}^{A}$ in one of the waveguides and mode $\mathrm{TE}_{10}^{s}$ in the other one.
5.3. Double Step between Misaligned Waveguides. In the third and last case study, two steps between the same waveguides are analyzed (see Figure 6(c)). In this case the notation associated with the number of modes used refers to the three waveguides, 34-10-34 being the modes selected in the three waveguides involved. In order to test the evanescent modes (i.e., those whose cut-off frequency is below the analyzed range of frequencies), a range of frequencies is selected where none of the modes in the smallest waveguide propagates (i.e., 90$95 \mathrm{GHz}$ ).

The thickness of waveguide $(s)$ is $0.3 \mathrm{~mm}$ to limit the attenuation of its first three pairs of modes to $3.9 \mathrm{~dB}, 10 \mathrm{~dB}$, and $11.5 \mathrm{~dB}$, respectively, having significant higher-order 


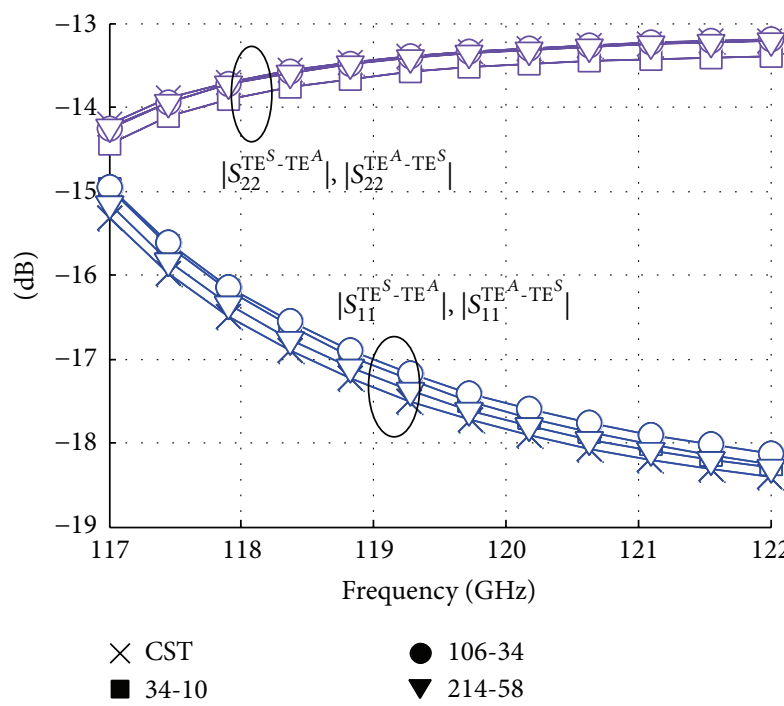

(a)



(c)

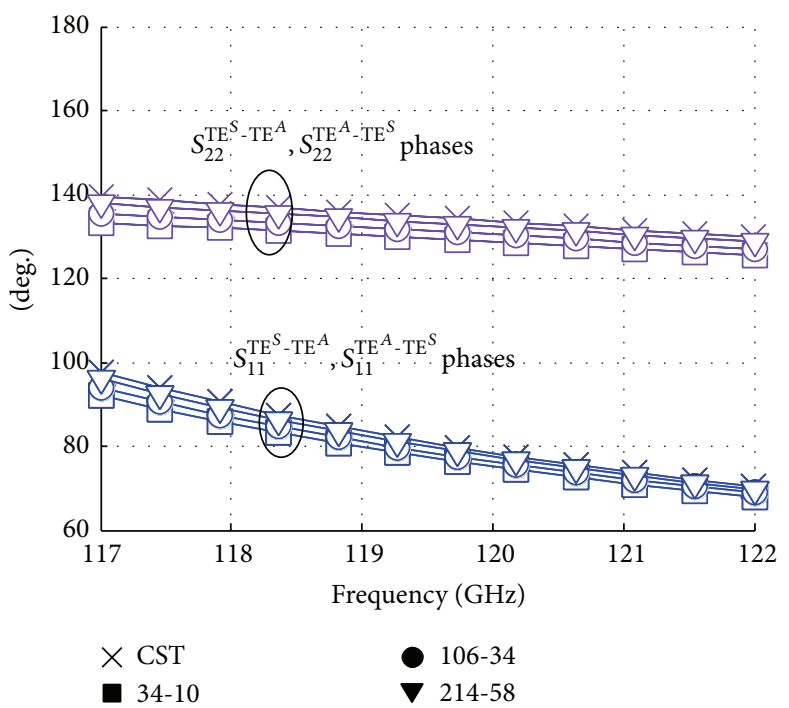

(b)

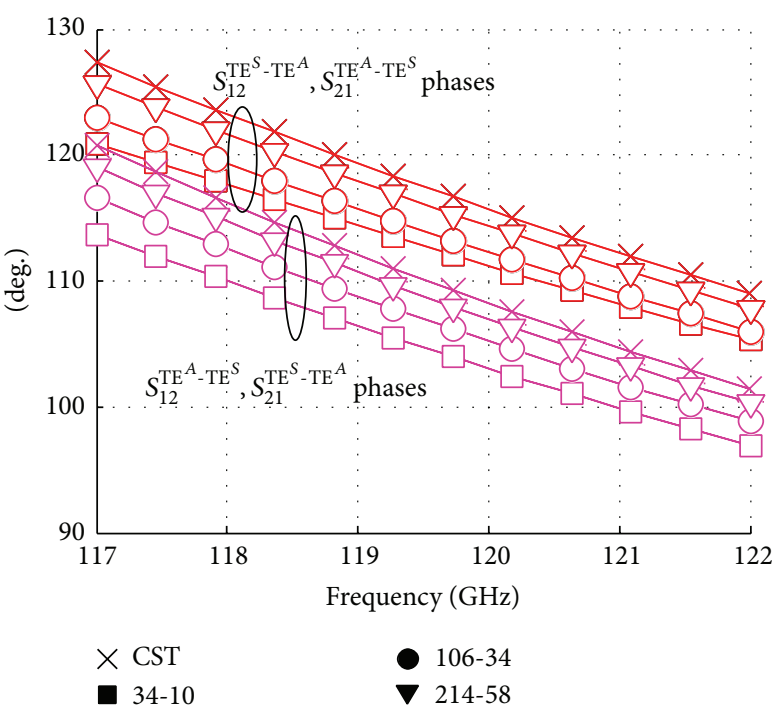

(d)

FIGURE 11: Case study b: mode $\mathrm{TE}_{10}^{A}$ in waveguide 1 to mode $\mathrm{TE}_{10}^{S}$ in waveguide 2 and mode $\mathrm{TE}_{10}^{S}$ in waveguide 1 to mode $\mathrm{TE}_{10}^{A}$ in waveguide 2: (a) reflection magnitude, (b) reflection phase, (c) transmission magnitude, and (d) transmission phase.

mode interaction between the two discontinuities. In Figures 12-14 it can be seen that all the results achieved using a very low number of modes (i.e., 34-10-34) and those with a higher number show greater differences than in the previous two cases. In any case, the results finally converge once the number of modes is increased.

\section{Conclusion}

The complete mathematical formulation of the ModeMatching method applied to equilateral triangular discontinuities has been presented. The mode spectrum for the equilateral triangular waveguide has been shown. A flexible formulation based on plane waves has been presented, simplifying the surface integrals involved. Moreover, this mathematical formulation can be easily extended to discontinuities that involve triangular equilateral waveguides with other classical waveguides such as the rectangular, circular, or elliptical waveguides, where their modes can be expanded in plane waves as well. Finally, three case studies have been analyzed and compared with the results obtained with the commercial software CST Microwave Studio. A good agreement between responses has been proved, with ModeMatching having the advantage of a quasi-analytical method with high efficiency. 




(a)

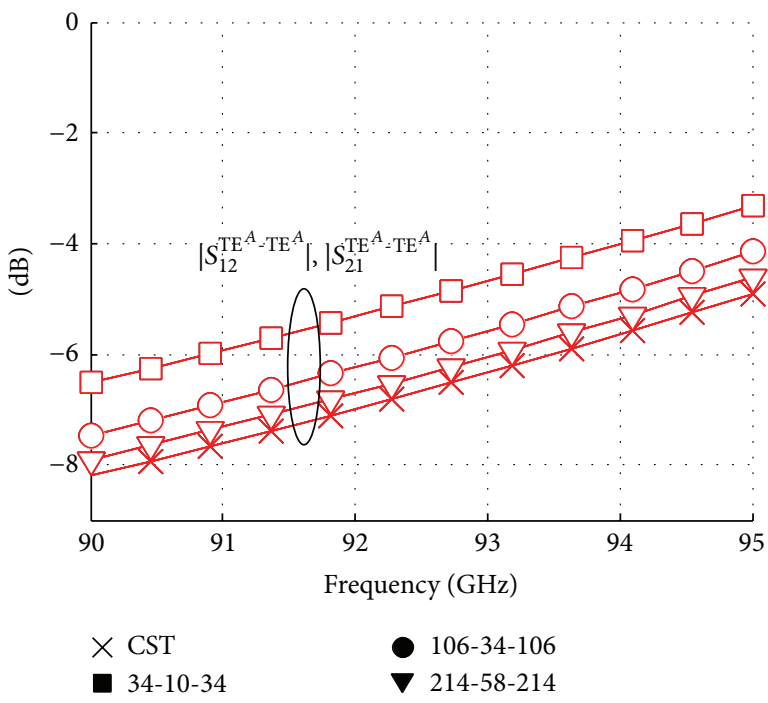

(c)



(b)

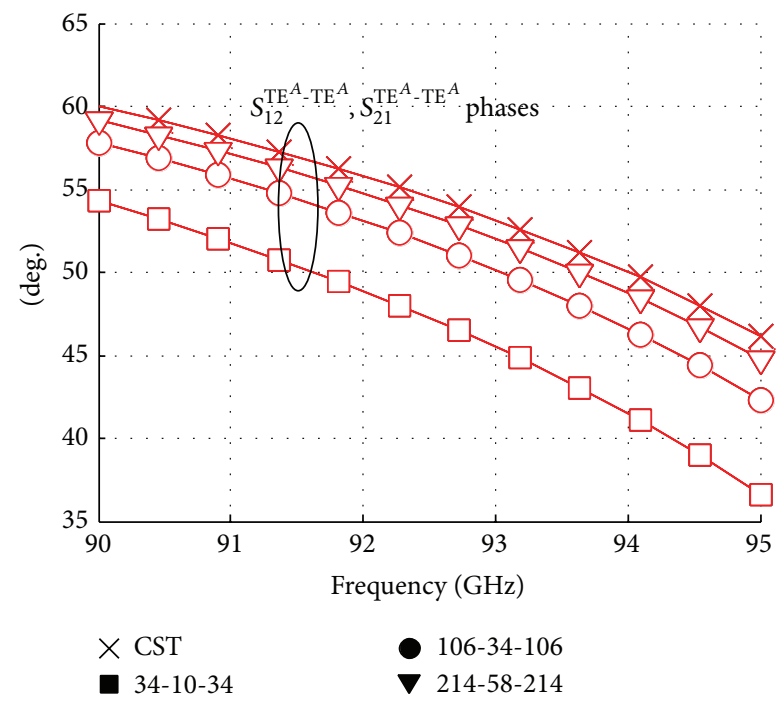

(d)

Figure 12: Case study c: mode $\mathrm{TE}_{10}^{A}$ in waveguide 1 to mode $\mathrm{TE}_{10}^{A}$ in waveguide 2: (a) reflection magnitude, (b) reflection phase, (c) transmission magnitude, and (d) transmission phase.

\section{Appendix}

\section{Mode Calculation}

The electromagnetic field of a TE mode, at the angular frequency $\omega=2 \pi f$, can be expressed using the following equations $[13,14]$ :

$$
\begin{aligned}
& h_{z p}=-\frac{\sqrt{Y_{p}} k_{c p}^{2}}{\gamma_{p}} \sqrt{N_{p}} \phi_{p}, \\
& \vec{h}_{p}=\sqrt{Y_{p}} \sqrt{N_{p}} \nabla_{t} \phi_{p},
\end{aligned}
$$

$$
\begin{aligned}
& e_{z p}=0, \\
& \vec{e}_{p}=\sqrt{Z_{p}} \sqrt{N_{p}} \nabla_{t} \phi_{p} \times \widehat{z},
\end{aligned}
$$

where the wave impedance (admittance) $Z_{p}\left(Y_{p}\right)$ is

$$
Z_{p}=Y_{p}^{-1}=\frac{j \omega \mu}{\gamma_{p}}
$$

and the normalization constant for unit complex power $N_{p}$ and the propagation constant $\gamma_{p}$ are

$$
\begin{aligned}
N_{p} & =\left[\iint_{S}\left|\nabla_{t} \phi_{p}\right|^{2} d s\right]^{-1}=\left[k_{c p}^{2} \iint_{S} \phi_{p}^{2} d s\right]^{-1}, \\
\gamma_{p}^{2} & =k_{c p}^{2}-\omega^{2} \mu \epsilon .
\end{aligned}
$$




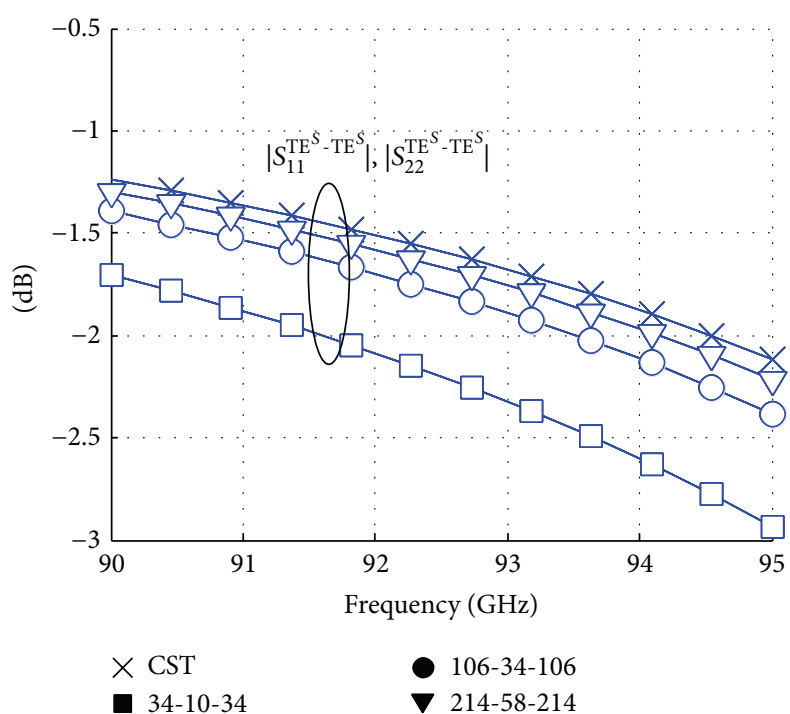

(a)

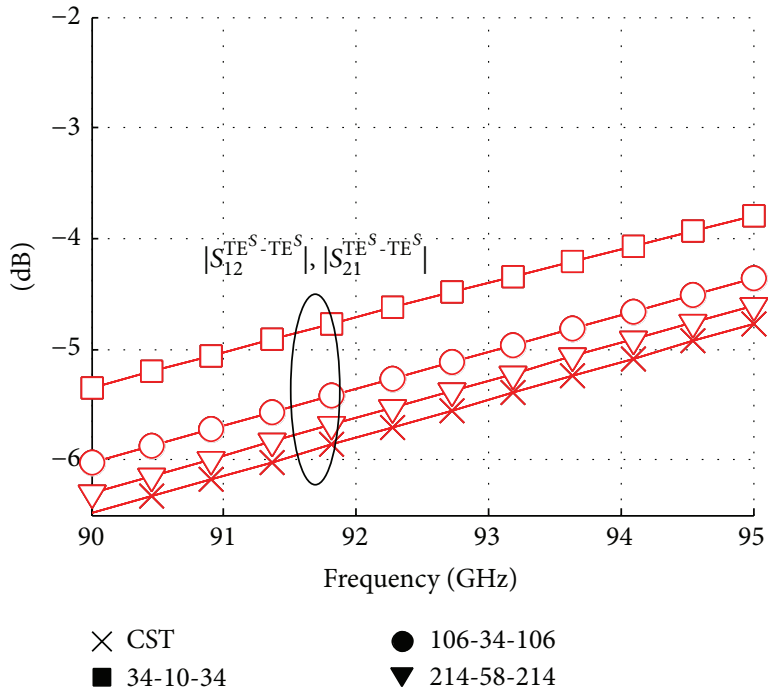

(c)

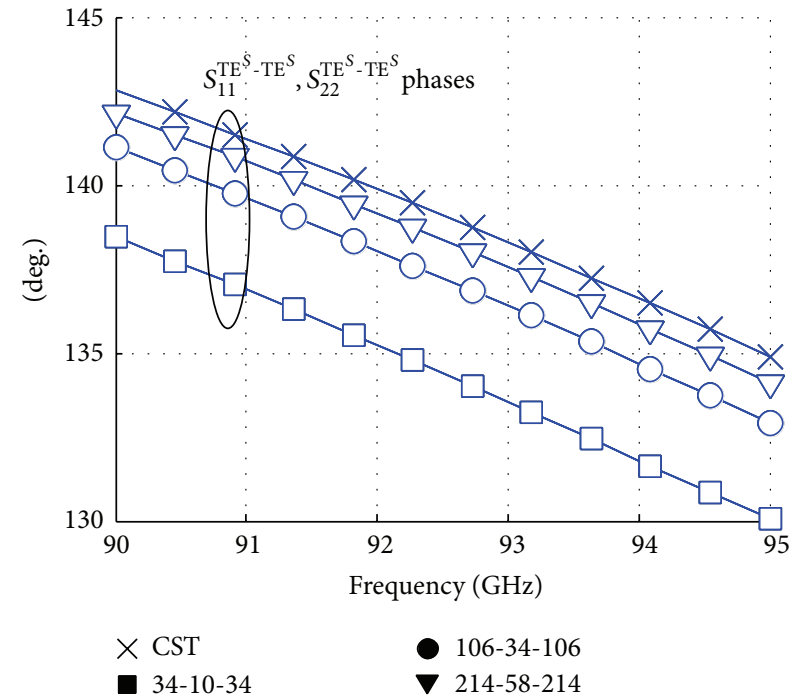

(b)

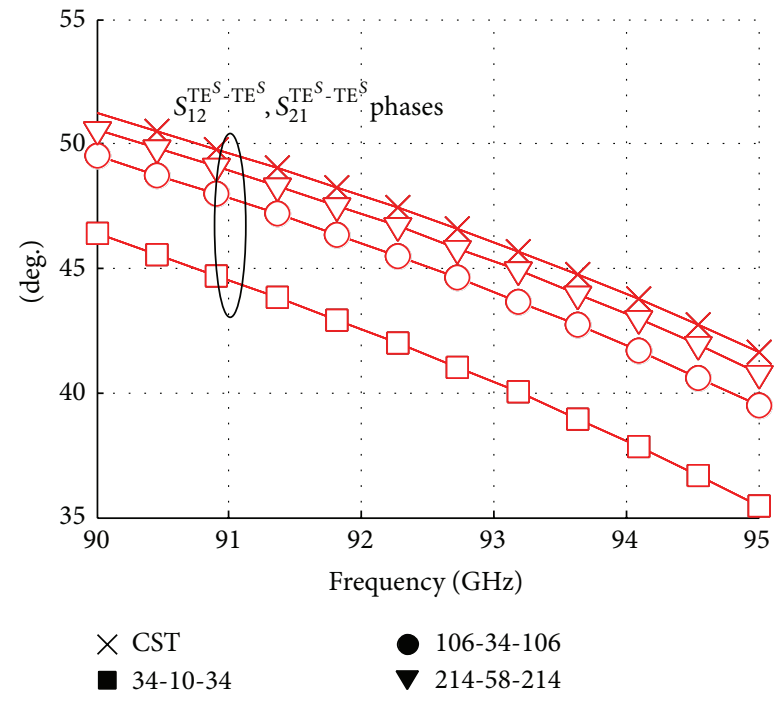

(d)

FIgure 13: Case study c: mode $\mathrm{TE}_{10}^{S}$ in waveguide 1 to mode $\mathrm{TE}_{10}^{S}$ in waveguide 2: (a) reflection magnitude, (b) reflection phase, (c) transmission magnitude, and (d) transmission phase.

The electromagnetic field of a TM mode can be expressed using the following equations:

$$
\begin{aligned}
& e_{z p}=-\frac{\sqrt{Z_{p}} k_{c p}^{2}}{\gamma_{p}} \sqrt{N_{p}} \phi_{p}, \\
& \vec{e}_{p}=\sqrt{Z_{p}} \sqrt{N_{p}} \nabla_{t} \phi_{p}, \\
& h_{z p}=0, \\
& \vec{h}_{p}=\sqrt{Y_{p}} \sqrt{N_{p}} \bar{z} \times \nabla_{t} \phi_{p},
\end{aligned}
$$

where the normalization constant for unit power $N_{p}$ and the wave number $k_{c p}$ hold the same definition as in (A.3a) and (A.3b), respectively, and the wave impedance (admittance) $Z_{p}\left(Y_{p}\right)$ is

$$
Z_{p}=Y_{p}^{-1}=\frac{\gamma_{p}}{j \omega \epsilon}
$$

\section{Competing Interests}

The authors declare that they have no competing interests. 


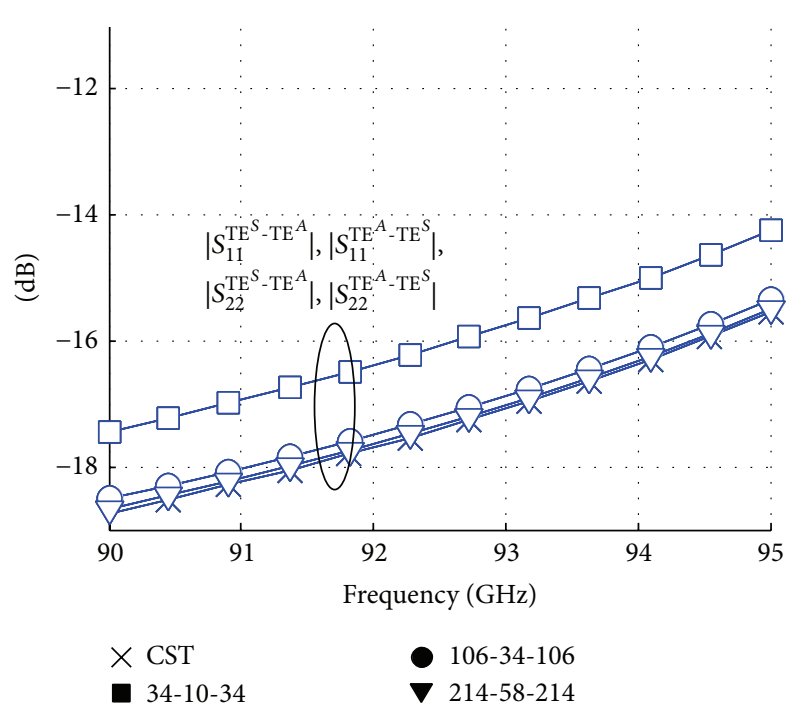

(a)

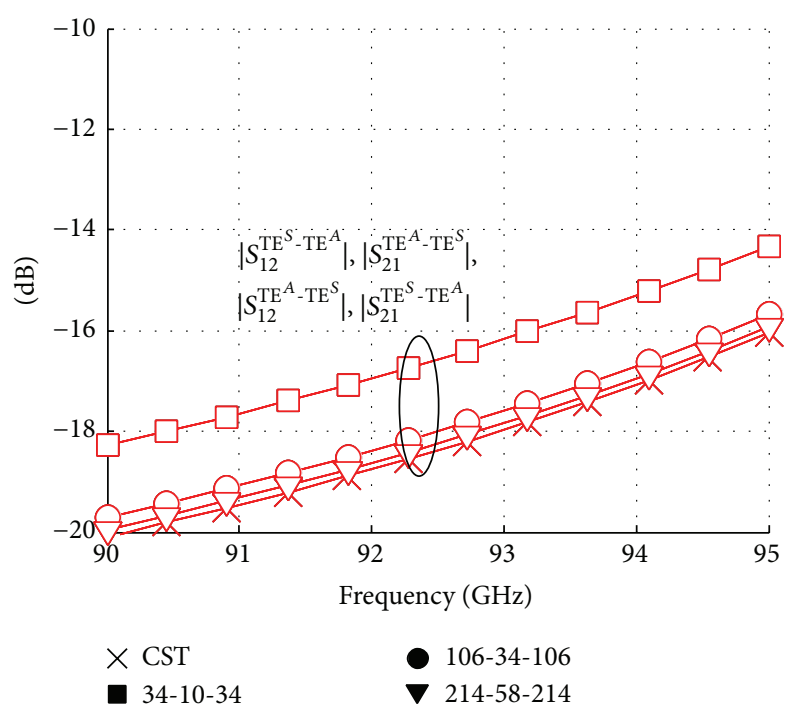

(c)

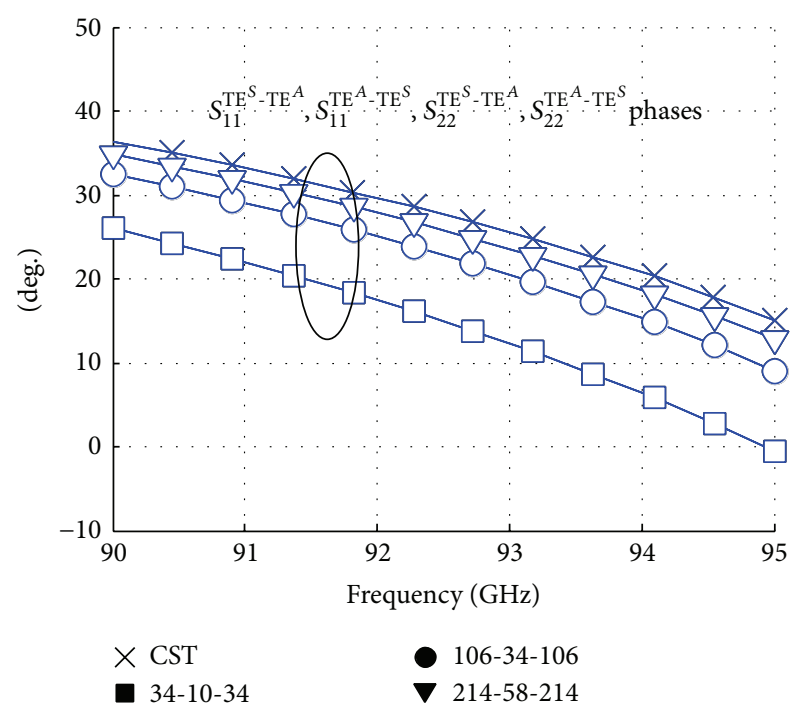

(b)

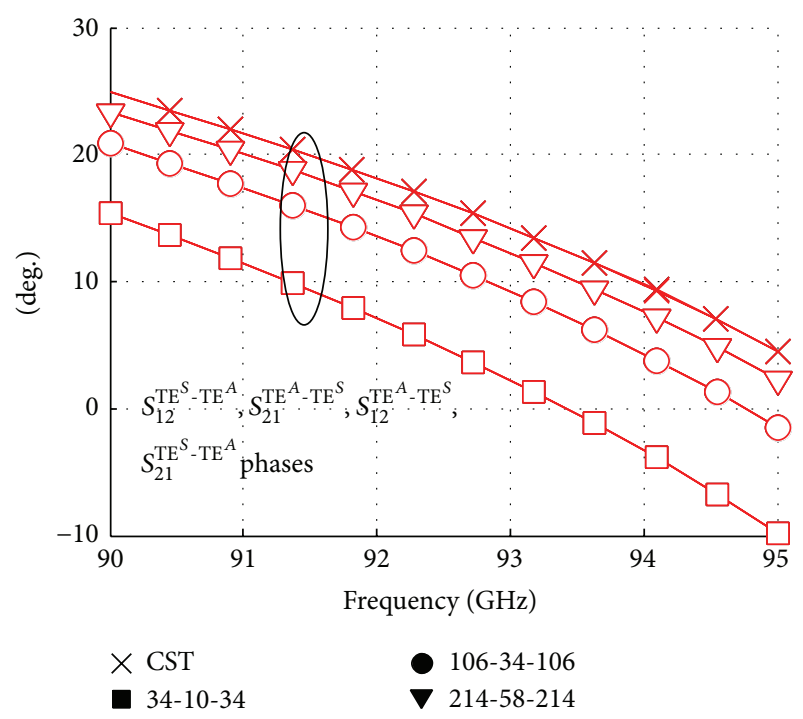

(d)

FIgUre 14: Case study c: mode $\mathrm{TE}_{10}^{A}$ in waveguide 1 to mode $\mathrm{TE}_{10}^{S}$ in waveguide 2 and mode $\mathrm{TE}_{10}^{S}$ in waveguide 1 to mode $\mathrm{TE}_{10}^{A}$ in waveguide 2: (a) reflection magnitude, (b) reflection phase, (c) transmission magnitude, and (d) transmission phase.

\section{Acknowledgments}

This work was supported by the Spanish government under Grant TEC2013-47106-C3-1/2-R and the Comunidad de Madrid Program S2013/ICE-3000 (SPADERADARCM).

\section{References}

[1] A. E. Grant and J. H. Meadows, Communication Technology Update and Fundamentals, Focal Press, Oxford, UK, 14th edition, 2014.

[2] D. Raychaudhuri and M. Gerla, Emerging Wireless Technologies and the Future Mobile Internet, Cambridge University Press, New York, NY, USA, 2011.

[3] G. A. Vandenbosch and A. Vasylchenko, A practical guide to 3D electromagnetic software tools, InTech, Rijeka, Croatia, 2011.
[4] Q. S. Cheng, J. W. Bandler, S. Koziel, M. H. Bakr, and S. Ogurtsov, "The state of the art of microwave CAD: EM-based optimization and modeling," International Journal of RF and Microwave Computer-Aided Engineering, vol. 20, no. 5, pp. 475491, 2010.

[5] A. A. Oliner, "Historical perspectives on microwave field theory," IEEE Transactions on Microwave Theory and Techniques, vol. 32, no. 9, pp. 1022-1045, 1984.

[6] A. Wexler, "Solution of waveguide discontinuities by modal analysis," IEEE Transactions on Microwave Theory and Techniques, vol. 15, no. 9, pp. 508-517, 1967.

[7] H. Patzelt and F. Arndt, "Double-plane steps in rectangular waveguides and their application for transformers, irises, and filters," IEEE Transactions on Microwave Theory and Techniques, vol. 30, no. 5, pp. 771-776, 1982. 
[8] J. A. Ruiz-Cruz, M. A. El Sabbagh, K. A. Zaki, J. M. Rebollar, and Y. Zhang, "Canonical ridge waveguide filters in LTCC or metallic resonators," IEEE Transactions on Microwave Theory and Techniques, vol. 53, no. 1, pp. 174-182, 2005.

[9] J. Esteban and J. M. Rebollar, "Generalized scattering matrix of generalized two-port discontinuities: application to four-port and nonsymmetric six-port couplers," IEEE Transactions on Microwave Theory and Techniques, vol. 39, no. 10, pp. 1725-1734, 1991.

[10] G. Conciauro, M. Guglielmi, and R. Sorrentino, Advanced Modal Analysis: CAD Techniques for Waveguide Components and Filters, John Wiley \& Sons, 1999.

[11] J. A. Ruiz-Cruz, J. M. Rebollar, and J. R. Montejo-Garai, Computer Aided Design of Waveguide Devices by Mode-Matching Methods, InTech, Rijeka, Croatia, 2010.

[12] P. M. Morse and H. Feshbach, Methods of Theoretical Physics, McGraw-Hill, New York, NY, USA, 1953.

[13] R. E. Collin, Field Theory of Guided Waves, IEEE Press, New York, NY, USA, 1991.

[14] D. M. Pozar, Microwave Engineering, John Wiley \& Sons, 1998.

[15] S. A. Schelkunoff, Electromagnetic Waves, van Nostrand, New York, NY, USA, 1943.

[16] P. L. Overfelt and D. J. White, "TE and TM modes of some triangular cross-section waveguides using superposition of plane waves," IEEE Transactions on Microwave Theory and Techniques, vol. 34, no. 1, pp. 161-167, 1986.

[17] B. J. McCartin, "Eigenstructure of the equilateral triangle, Part I: the Dirichlet problem," SIAM Review, vol. 45, no. 2, pp. 267-287, 2003.

[18] B. J. McCartin, "Eigenstructure of the equilateral triangle, part II: the Neumann problem," Mathematical Problems in Engineering, vol. 8, no. 6, pp. 517-539, 2002.

[19] T. Itoh, Numerical Techniques for Microwave and MillimeterWave Passive Structures, John Wiley \& Sons, New York, NY, USA, 1989.

[20] J. Córcoles, M. A. González De Aza, and J. Zapata, "Full-wave analysis of finite periodic cylindrical conformal arrays with Floquet spherical modes and a hybrid finite element -generalized scattering matrix method," Journal of Electromagnetic Waves and Applications, vol. 28, no. 1, pp. 102-111, 2014.

[21] J. Rubio, J. Corcoles, and M. Gonzalez de Aza, "Inclusion of the feeding network effects in the generalized-scattering-matrix formulation of a finite array," IEEE Antennas and Wireless Propagation Letters, vol. 8, pp. 819-822, 2009.

[22] G. Figlia and G. G. Gentili, "On the line-integral formulation of mode-matching technique," IEEE Transactions on Microwave Theory and Techniques, vol. 50, no. 2, pp. 578-580, 2002. 


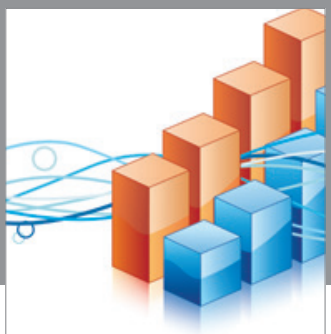

Advances in

Operations Research

vatem alat4

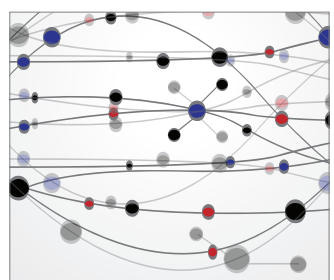

\section{The Scientific} World Journal
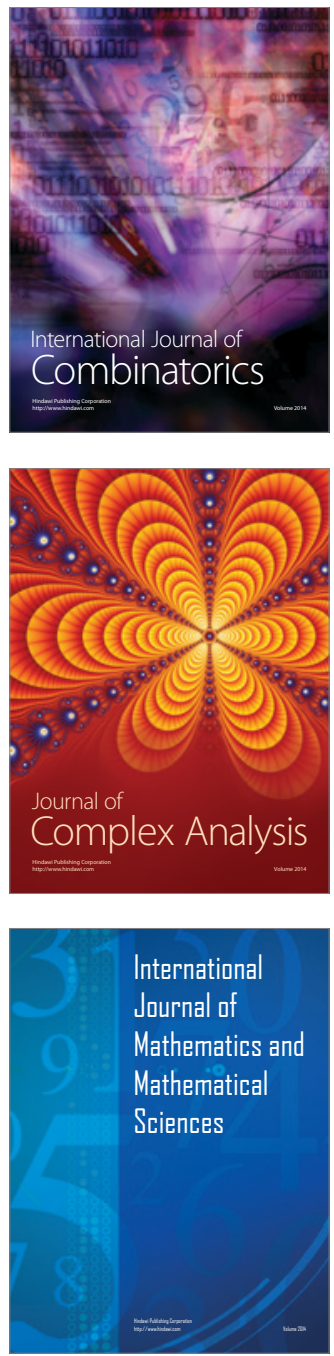
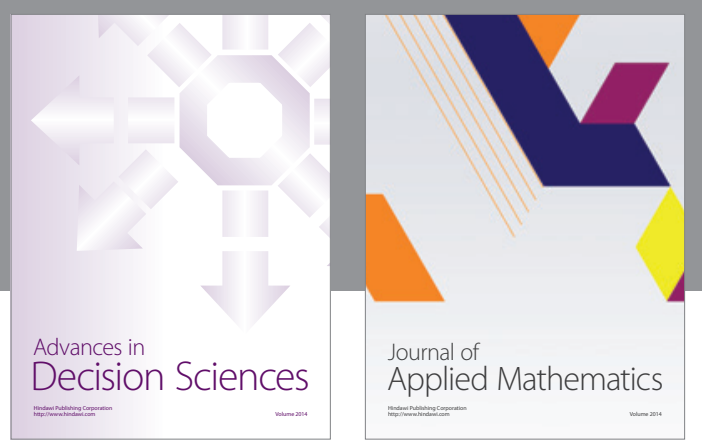

Algebra

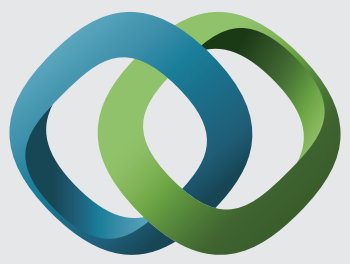

\section{Hindawi}

Submit your manuscripts at

http://www.hindawi.com
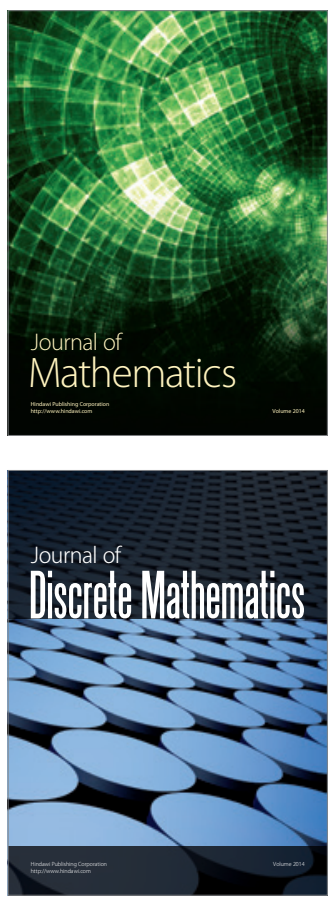

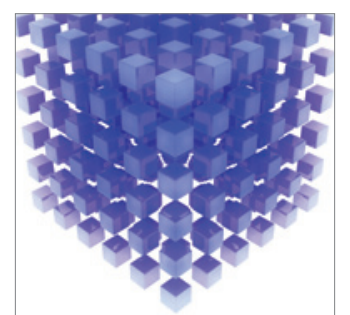

Mathematical Problems in Engineering
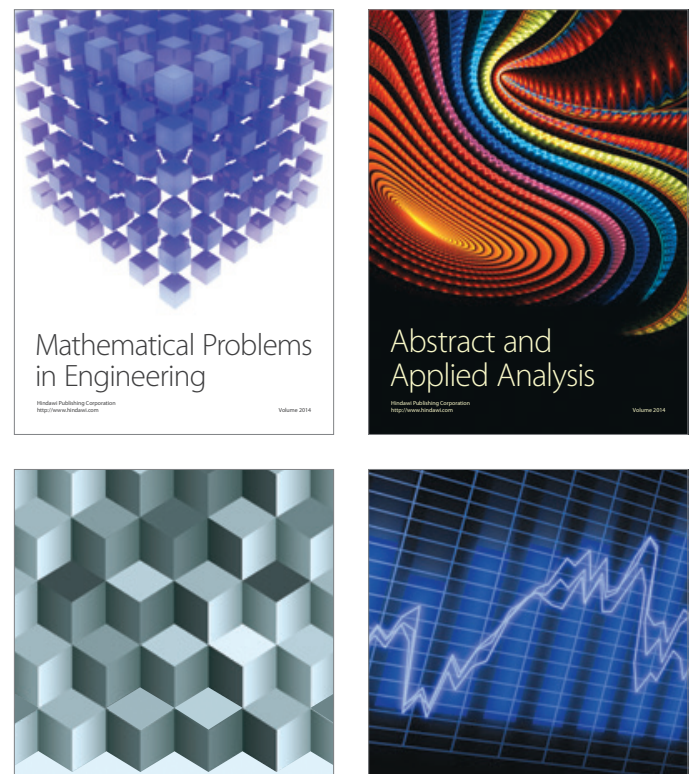

Journal of

Function Spaces

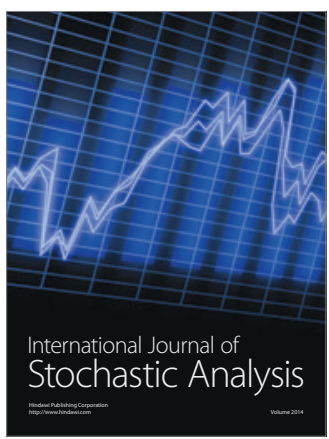

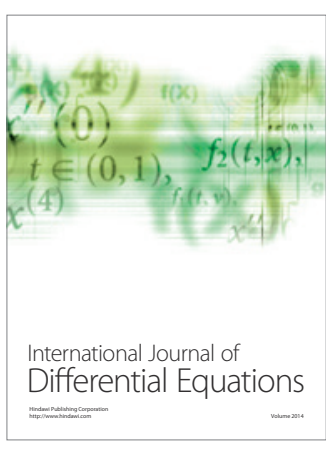
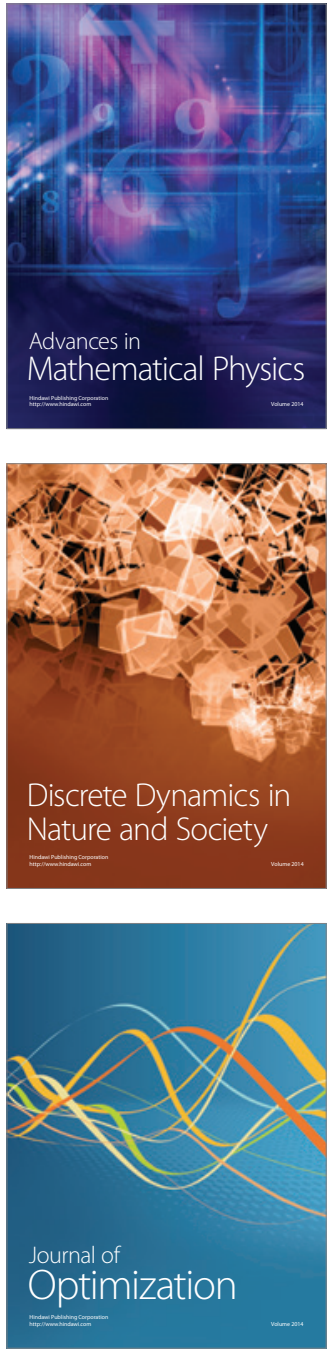